\title{
Experimental constraints on the thermodynamics and sound velocities of hcp-Fe to core pressures
}

\author{
Caitlin A. Murphy, ${ }^{1}$ Jennifer M. Jackson, ${ }^{1}$ and Wolfgang Sturhahn ${ }^{1}$ \\ Received 27 November 2012; revised 7 March 2013; accepted 19 March 2013; published 20 May 2013.
}

[1] We report the high-pressure thermoelastic and vibrational thermodynamic parameters for hexagonal close-packed iron $(\varepsilon-\mathrm{Fe})$, based on nuclear resonant inelastic X-ray scattering and in situ X-ray diffraction experiments at $300 \mathrm{~K}$. Long data collection times, high-energy resolution, and quasi-hydrostatic sample conditions produced a high-statistical quality data set that comprises the volume-dependent phonon density of states (DOS) of $\varepsilon$-Fe at eleven compression points. From the integrated phonon DOS, we determine the Lamb-Mössbauer factor $\left(f_{\mathrm{LM}}\right)$, average force constant $(\Phi)$, and vibrational entropy $\left(S_{\mathrm{vib}}\right)$ of $\varepsilon$-Fe to pressures relevant to Earth's outer core. We find $f_{\mathrm{LM}}=0.923 \pm 0.001$ at $171 \mathrm{GPa}$, suggesting restricted thermal atomic motion at large compressions. We use $\Phi$ to approximate $\varepsilon-\mathrm{Fe}$ 's pressure- and temperature-dependent reduced isotopic partition function ratios ( $\beta$-factors), which provide information about the partitioning behavior of iron isotopes in equilibrium processes involving solid $\varepsilon$-Fe. In addition, we use the volume dependence of $S_{\text {vib }}$ to determine the product of $\varepsilon$-Fe's vibrational thermal expansion coefficient and isothermal bulk modulus, which we find to be pressure-independent and equal to $5.70 \pm 0.05 \mathrm{MPa} / \mathrm{K}$ at $300 \mathrm{~K}$. Finally, from the low-energy region of each phonon DOS, we determine the Debye sound velocity $\left(v_{\mathrm{D}}\right)$, from which we derive the compressional $\left(v_{\mathrm{P}}\right)$ and shear $\left(v_{\mathrm{S}}\right)$ sound velocities of $\varepsilon$-Fe. We find $v_{\mathrm{D}}=5.60 \pm 0.06, v_{\mathrm{P}}=10.11 \pm 0.12$, and $v_{\mathrm{S}}=4.99 \pm 0.06 \mathrm{~km} / \mathrm{s}$ at $171 \mathrm{GPa}$, thus providing a new tight constraint on the density dependence of $\varepsilon-\mathrm{Fe}$ 's sound velocities to outer core pressures.

Citation: Murphy, C. A., J. M. Jackson, and W. Sturhahn (2013), Experimental constraints on the thermodynamics and sound velocities of hcp-Fe to core pressures, J. Geophys. Res. Solid Earth, 118, 1999-2016, doi:10.1002/jgrb.50166.

\section{Introduction}

[2] The Earth's core is thought to be made up of iron, nickel, and a few percent light elements [e.g., McDonough, 2003]. Iron is considered to be the most abundant element in the core, and existing data suggest that it crystallizes in the hexagonal close-packed (hcp) structure $(\varepsilon-\mathrm{Fe})$ at core conditions [e.g., Mao et al., 1990; Alfè et al., 2001; Ma et al., 2004; Dewaele et al., 2006; Tateno et al., 2010]. In turn, a large theoretical and experimental effort has been dedicated to determining $\varepsilon$-Fe's material properties at highpressure and temperature $(P T)$ conditions.

[3] Many studies have focused on properties of $\varepsilon$-Fe that can also be inferred for Earth's solid inner core via seismology, which is the most direct tool available for probing the deep Earth. For example, seismologists have provided onedimensional models for the inner core's compressional and shear sound velocities and density based on seismic wave

\footnotetext{
${ }^{1}$ Seismological Laboratory, California Institute of Technology, Pasadena, California, USA.

Corresponding author: C. A. Murphy, Seismological Laboratory, California Institute of Technology, M/C 252-21, Pasadena, CA 91125 , USA. (caitlinm@caltech.edu)

(C)2013. American Geophysical Union. All Rights Reserved. 2169-9313/13/10.1002/jgrb.50166
}

travel times and normal modes from thousands of seismic events [e.g., Dziewonski and Anderson, 1981; Kennett et al., 1995]. Many experimental and theoretical studies have thus explored $\varepsilon$-Fe's equation of state in general [e.g., Brown and McQueen, 1986; Mao et al., 1990; Wasserman et al., 1996; Dubrovinsky et al., 1998; Dewaele et al., 2006; Sola et al., 2009; Sha and Cohen, 2010] and, in particular, its sound velocities [e.g., Brown and McQueen, 1986; Mao et al., 2001; Nguyen and Holmes, 2004; Lin et al., 2005; Mao et al., 2008; Sha and Cohen, 2010; Antonangeli et al., 2012] and thermal expansion coefficient [e.g., Jeanloz, 1979; Boehler et al., 1990; Duffy and Ahrens, 1993; Wasserman et al., 1996; Stixrude et al., 1997; Dubrovinsky et al., 2000; Alfè et al., 2001; Anderson et al., 2001; Isaak and Anderson, 2003; Sha and Cohen, 2010] to the pressures expected to be found in Earth's core.

[4] Results from such experiments are often compared with both seismic observations and reported properties of iron alloys, in an attempt to better constrain the composition of Earth's core. However, significant uncertainties remain due to technical difficulties associated with inferring thermoelastic properties of the remote inner core from seismic observations [e.g., Shearer and Masters, 1990; Cao and Romanowicz, 2004], discrepancies among theoretical calculations [e.g., Steinle-Neumann et al., 2004; Vočadlo et al., 2009], and the statistical quality and accessible $P T$ conditions of experimental studies. 
MURPHY ET AL.: THERMOELASTICITY OF hcp-Fe TO $171 \mathrm{GPa}$

Table 1. Vibrational Thermodynamic Parameters of $\varepsilon$-Fe From the Phonon DOS

\begin{tabular}{|c|c|c|c|c|c|c|}
\hline$V\left(\mathrm{~cm}^{3} / \mathrm{mol}\right)^{\mathrm{a}}$ & $P(\mathrm{GPa})^{\mathrm{a}}$ & $f_{\mathrm{LM}}{ }^{\mathrm{c}}$ & $\Phi(\mathrm{N} / \mathrm{m})^{\mathrm{c}}$ & $10^{3} \ln \beta_{57 \mathrm{Fe} / 54 \mathrm{Fe}}{ }^{\mathrm{d}}$ & $S_{\text {vib }}\left(k_{\mathrm{B}} / \text { atom }\right)^{\mathrm{c}}$ & $\alpha_{\mathrm{vib}}\left(10^{-5} \mathrm{~K}^{-1}\right)^{\mathrm{e}}$ \\
\hline $5.92(2)^{\mathrm{b}}$ & $30(2)$ & $0.857(1)$ & $239(6)$ & $11.4(3)$ & $2.63(2)$ & $1.84(2)$ \\
\hline $5.81(1)^{\mathrm{b}}$ & $36(2)$ & $0.862(2)$ & $255(12)$ & $12.1(6)$ & $2.57(3)$ & $1.69(2)$ \\
\hline $5.56(1)^{\mathrm{b}}$ & $53(2)$ & $0.876(2)$ & $302(10)$ & $14.3(5)$ & $2.38(3)$ & $1.40(2)$ \\
\hline $5.36(1)^{\mathrm{b}}$ & $69(3)$ & $0.888(1)$ & $343(9)$ & $16.3(4)$ & $2.24(2)$ & $1.20(2)$ \\
\hline $5.27(2)$ & $77(3)$ & $0.892(1)$ & $356(5)$ & $16.9(3)$ & $2.20(1)$ & $1.13(2)$ \\
\hline $5.15(2)$ & $90(3)$ & $0.899(1)$ & $382(8)$ & $18.2(4)$ & $2.10(1)$ & $1.03(2)$ \\
\hline $5.00(2)^{\mathrm{f}}$ & $106(3)$ & $0.904(1)$ & $417(10)$ & $19.8(5)$ & $2.01(1)$ & $0.92(2)$ \\
\hline $4.89(2)^{\mathrm{f}}$ & $121(3)$ & $0.910(1)$ & $451(8)$ & $21.5(4)$ & $1.92(1)$ & $0.85(2)$ \\
\hline $4.81(2)^{\mathrm{f}}$ & $133(4)$ & $0.913(1)$ & $473(9)$ & $22.5(4)$ & $1.87(2)$ & $0.79(2)$ \\
\hline $4.70(2)^{\mathrm{f}}$ & $151(5)$ & $0.918(1)$ & $494(10)$ & $23.5(5)$ & $1.81(2)$ & $0.73(2)$ \\
\hline $4.58(2)^{\mathrm{f}}$ & $171(5)$ & $0.923(1)$ & $545(15)$ & $25.9(7)$ & $1.70(2)$ & $0.67(2)$ \\
\hline
\end{tabular}

${ }^{\mathrm{a}}$ Molar volumes per ${ }^{57} \mathrm{Fe}$ atom $(V)$ were measured with in situ XRD and converted to pressures $(P)$ using the Vinet EOS reported by Dewaele et al. [2006]. We note that reported volume errors are identical to those in our previous related studies [Murphy et al., 2011b, 2011a]. Pressure errors account for correlation between EOS parameters based on our fitting of the XRD data reported by Dewaele et al. [2006], and are therefore smaller than our previously reported values, which assumed no correlation.

${ }^{\mathrm{b}}$ For these measurements, neon was loaded as the pressure transmitting medium. For all other compression points, the sample was fully embedded in a boron epoxy insert.

${ }^{\mathrm{c}}$ The Lamb-Mössbauer factor $\left(f_{\mathrm{LM}}\right)$, average force constant acting on the resonant isotope in the lattice $(\Phi)$, and vibrational entropy $\left(S_{\mathrm{vib}}\right)$ per ${ }^{57} \mathrm{Fe}$ atom were determined from the integrated phonon DOS (equations (1), (2), and (9)). Values in parentheses give uncertainties for the last significant digit(s) reported, as determined by the PHOENIX software [Sturhahn, 2000].

${ }^{\mathrm{d}}$ Reduced isotopic partition function ratios $\left(10^{3} \ln \beta_{57 \mathrm{Fe} / 54 \mathrm{Fe}}\right)$ for $\varepsilon$-Fe at $300 \mathrm{~K}$ are based on our $\Phi$ (equation (2)) and the procedure described in section 4.1 ; uncertainties in the last significant digit reflect uncertainties in $\Phi$ as determined by the PHOENIX software [Sturhahn, 2000].

${ }^{\mathrm{e}}$ The vibrational component of the thermal expansion coefficient $\left(\alpha_{\mathrm{vib}}\right)$ for $\varepsilon$-Fe at $300 \mathrm{~K}$ is determined from our $S_{\mathrm{vib}}(V)($ equation $(9))$ and the Vinet EOS parameters reported by Dewaele et al. [2006], as described in section 5; uncertainties in the last significant digit reflect the uncertainties from an errorsweighted least-squares linear fit of our $S_{\mathrm{vib}}(V)$, with uncertainties in $S_{\mathrm{vib}}$ determined by the PHOENIX software [Sturhahn, 2000].

${ }^{\mathrm{f}}$ Texturing was observed at these compression points in the form of a loss of intensity in the (002) diffraction peak (section 2).

[5] The purpose of this study is to report the high-pressure thermoelastic and vibrational thermodynamic properties of $\varepsilon$-Fe, based on high-statistical quality nuclear resonant inelastic X-ray scattering (NRIXS) and in situ X-ray diffraction (XRD) experiments. NRIXS probes the partial projected phonon density of states (DOS) of materials containing select resonant isotopes such as ${ }^{57} \mathrm{Fe}$ [Sturhahn et al., 1995; Sturhahn, 2004; Sturhahn and Jackson, 2007], and in the case of pure ${ }^{57} \mathrm{Fe}$, NRIXS probes the total phonon DOS. We previously used this technique with in situ XRD to measure the volume-dependent total phonon DOS of $\varepsilon$-Fe at eleven compression points between 30 and $171 \mathrm{GPa}$ at $300 \mathrm{~K}$ [Murphy et al., 2011b, 2011a]. From the resulting data set, we determined $\varepsilon$-Fe's vibrational Grüneisen parameter [Murphy et al., 2011a], thermal atomic motion, and thermal pressure [Murphy et al., 2011b] to core pressures. These parameters allowed us to constrain the temperature at the inner core boundary via a prediction of the melting behavior of $\varepsilon-\mathrm{Fe}$, as well as the core-density deficit, or the amount of light elements that must be present in Earth's solid inner core to match seismic observations.

[6] In this study, we use the same data set to determine additional thermoelastic and vibrational thermodynamic parameters for $\varepsilon$-Fe, some of which are closely related to properties inferred from seismology. Furthermore, we report additional thermodynamic parameters based on $\varepsilon$-Fe's lattice dynamics that offer insights into isotopic partitioning of iron during equilibrium processes involving solid $\varepsilon$-Fe. Details for obtaining each parameter from the phonon DOS will be presented in their respective sections, in addition to a discussion of their potential applications in the context of Earth's core.

\section{Experiments}

[7] The present analysis is performed on the NRIXS and in situ XRD data set presented by Murphy et al. [2011a, 2011b]. For these experiments, we used modified panoramic diamond anvil cells (DACs) with $90^{\circ}$ openings and cubic boron nitride backing plates on the downstream side, thus maximizing the range of available diffraction angles for in situ XRD. Three DACs were prepared in total using beveled anvils with flat culet diameters of 250 or $150 \mu \mathrm{m}$, beryllium gaskets, and boron epoxy inserts that stabilized the gasket hole during compression. A neon pressure transmitting medium surrounded a piece of $10 \mu \mathrm{m}$ thick $95 \%$ isotopically enriched ${ }^{57} \mathrm{Fe}$ foil for our four smallest compression points, resulting in quasi-hydrostatic sample conditions to a pressure of $69 \mathrm{GPa}$ (Table 1). For all other compression points, the ${ }^{57} \mathrm{Fe}$ foil was fully embedded in the boron epoxy.

[8] Our NRIXS and in situ XRD experiments were performed at Sector 3-ID-B of the Advanced Photon Source at Argonne National Laboratory, with an average energy resolution of $1.2 \mathrm{meV}$ at the full-width half-maximum [Toellner, 2000]. A typical NRIXS scan consisted of tuning the highresolution monochromator from -65 to $+85 \mathrm{meV}$ around the nuclear resonance energy of ${ }^{57} \mathrm{Fe}(14.4125 \mathrm{keV})$, with a step size of $0.25 \mathrm{meV}$ and a collection time of $5 \mathrm{~s}$ per energy step. Vibrational information for $\varepsilon$-Fe extends to higher energies with compression, so we increased this range to -70 to $+90 \mathrm{meV}$ for our two largest compression points. Between 8 and 21 NRIXS scans were collected for each compression point, with the exception of our measurement at $5.81 \pm 0.01 \mathrm{~cm}^{3} / \mathrm{mol}(P=36 \pm 2 \mathrm{GPa})$, for which only four scans were collected. NRIXS data were analyzed with the PHOENIX software [Sturhahn, 2000] (http://www.nrixs.com), which was used to remove the elastic contribution and apply the quasi-harmonic lattice model, in order to obtain $\varepsilon$-Fe's total phonon DOS, $D(E, V)$.

[9] X-ray diffraction data were collected in situ before and after each NRIXS data set at the same sample position, to determine the atomic volume at each compression point (Table 1). We observed some texturing in our five largest 


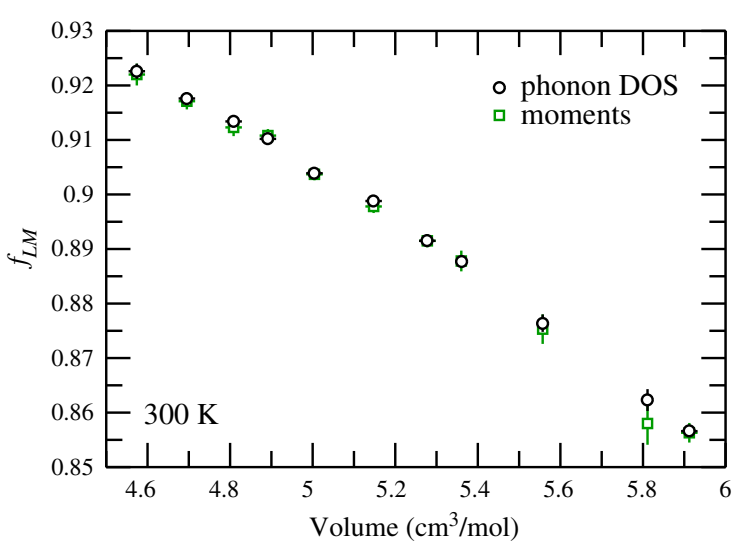

Figure 1. Lamb-Mössbauer factor of $\varepsilon$-Fe from NRIXS data. Black circles give the Lamb-Mössbauer factor $\left(f_{\mathrm{LM}}\right)$ as determined from $\varepsilon$-Fe's total phonon DOS and equation (1); green squares show $f_{\mathrm{LM}}$ as determined from the zeroth-order moment of $S(E)$ (section 3).

compression points, as indicated by a loss of intensity in the (002) diffraction peak, which likely resulted from nonhydrostatic conditions at extreme pressures. The remaining diffraction peaks were sufficient for determining sample volumes at our five largest compression points, because the (100) peak is sensitive to the $a$ unit cell parameter, while the (101) peak is sensitive to both the $a$ and $c$ unit cell parameters in an hcp unit cell. We note that the $c / a$ ratios for our five largest compression points are consistent with the trend suggested by our smaller compression points, where three XRD peaks are available. The exact amount of additional uncertainty associated with determining the sample volume from fewer diffraction peaks is difficult to quantify, so we report volume uncertainties that are twice as large as the determined uncertainties of our unit cell parameters in Table 1.

[10] For our determination of the phonon DOS and data analysis, we relied on our in situ measured volumes. To present our results on a common scale and for discussion, we convert our measured volumes to pressures $(P)$ using the Vinet equation of state (EOS) for $\varepsilon-F e$ reported by Dewaele et al. [2006]. For comparison, we note that the pressures predicted by the third-order Birch-Murnaghan (BM) EOS for $\varepsilon$-Fe reported by Mao et al. [1990] are identical to those given in Table 1 within uncertainty, with the exception of our largest compression point: the Vinet EOS reported by Dewaele et al. [2006] indicates a pressure of $171 \mathrm{GPa}$, while the BM EOS reported by Mao et al. [1990] indicates a pressure of $178 \mathrm{GPa}$.

\section{Lamb-Mössbauer Factor and Its Relation to the Melting Behavior of $\varepsilon-F e$}

[11] The Lamb-Mössbauer factor $\left(f_{\mathrm{LM}}\right)$ represents the probability for recoilless absorption, or the ratio of elastic to total incoherent scattering in NRIXS experiments. It has a similar functional form as that of the Debye-Waller factor $\left(f_{\mathrm{DW}}\right)$, where $f_{\mathrm{DW}}$ describes coherent, fast scattering events and $f_{\mathrm{LM}}$ describes slow scattering events, i.e., events that occur over the lifetime of nuclear resonance (141 ns for ${ }^{57} \mathrm{Fe}$ ) [Sturhahn, 2004]. In general, $f_{\mathrm{LM}}$ can best be understood by its relationship to the thermal motion of resonant nuclei about their equilibrium positions: $f_{\mathrm{LM}}=\exp \left[-k_{0}^{2}\left\langle u^{2}\right\rangle\right]$, where $k_{0}$ is the wave number of the resonant X-rays $\left(7.306 \AA^{-1}\right.$ for $\left.{ }^{57} \mathrm{Fe}\right)$ and $<u^{2}>$ is the mean-square atomic displacement. This relationship highlights the fact that $f_{\mathrm{LM}}$ contains information about lattice dynamics and, in turn, depends strongly on the binding of the resonant nuclei in the lattice (e.g., on composition, crystal structure, and $P T$ conditions).

[12] There are two ways to access $f_{\mathrm{LM}}$ from NRIXS data. First, $f_{\mathrm{LM}}$ can be determined from an analysis of the zeroth-order moment of the excitation probability density, $S(E)$, which is extracted from the raw NRIXS data by fitting and subtracting the elastic peak and performing the proper normalization and refinement procedures [Sturhahn et al., 1995; Sturhahn, 2000]. Then, $f_{\mathrm{LM}}$ is obtained by taking the zeroth-order moment of $S(E)$, or $S_{n}=\int E^{n} S(E) \mathrm{d} E$ for $n=0$. Details of this procedure were previously presented by Sturhahn and Chumakov [1999].

[13] The second method for obtaining $f_{\mathrm{LM}}$ from NRIXS data is via the measured total phonon DOS, $D(E, V)$, which we obtain by applying a quasi-harmonic oscillator (lattice) model to the total $S(E)$ described above [Sturhahn, 2000]. In particular,

$$
f_{\mathrm{LM}}(V)=\exp \left[-\int \frac{E_{R}}{E} \operatorname{coth}\left(\frac{E}{2 k_{B} T}\right) D(E, V) d E\right],
$$

where $k_{\mathrm{B}}$ is Boltzmann's constant and the phonon DOS has been normalized by $\int D(E) \mathrm{d} E=3$ [Sturhahn and Jackson, 2007]. Values for $f_{\mathrm{LM}}$ determined using equation (1) are given in Table 1. To demonstrate the high-statistical quality of our data set, we compare our uncertainties for $f_{\mathrm{LM}}$ with those measured by Mao et al. [2001] up to $153 \mathrm{GPa}$ : performing the same PHOENIX analysis on both data sets, we find that our data produce errors in $f_{\mathrm{LM}}$ that are $\sim 75 \%$ smaller on average using the moments method, and $\sim 60 \%$ smaller using equation (1).

[14] Values for $f_{\mathrm{LM}}$ determined using the two described methods are indistinguishable for all of our compression points, as can be seen in Figure 1. The largest discrepancy occurs at a molar volume per ${ }^{57} \mathrm{Fe}$ atom of $5.81 \pm 0.01 \mathrm{~cm}^{3} / \mathrm{mol}$ $(P=36 \pm 2 \mathrm{GPa})$, which is the compression point at which we had the lowest overall counts because of fewer scans collected (section 2). Such disagreement demonstrates the importance of a high-statistical quality data set for accurately determining vibrational information. We note that the two methods for obtaining $f_{\mathrm{LM}}$ are related by $S(E)$, but the moments analysis method requires no assumptions, while the use of equation (1) assumes that a quasi-harmonic oscillator model accurately describes the behavior of $\varepsilon-\mathrm{Fe}$ [e.g., Sturhahn, 2004]. Good agreement between $f_{\mathrm{LM}}$ determined from the two distinct methods is consistent with the validity of this model over our experimental $P T$ conditions.

[15] As previously stated, $f_{\mathrm{LM}}$ can best be understood in the context of lattice dynamics by considering the relationship $f_{\mathrm{LM}}=\exp \left[-k_{0}^{2}\left\langle u^{2}\right\rangle\right]$. From this relationship, it is evident that an increase in $f_{\mathrm{LM}}$ with compression corresponds to a reduction of thermal motion-i.e., reduced displacement of the iron atoms, or stiffening of the lattice-or a decrease in $\left\langle u^{2}\right\rangle$. We have previously used this behavior to predict $\varepsilon$-Fe's melting curve shape based on Gilvarry's reformulation of Lindemann's melting criterion [Gilvarry, 
MURPHY ET AL.: THERMOELASTICITY OF hep-Fe TO $171 \mathrm{GPa}$

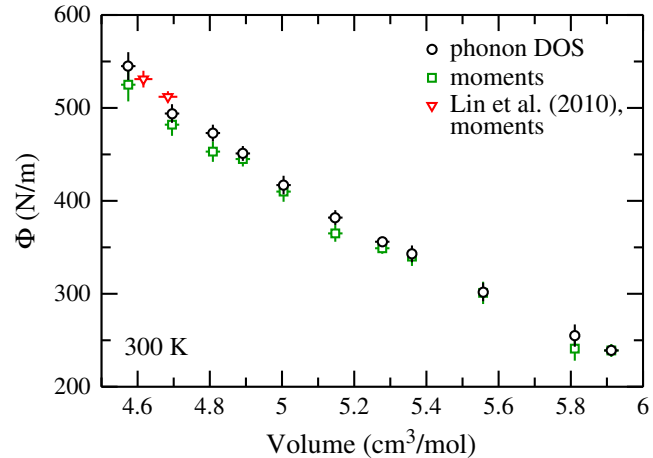

(a)

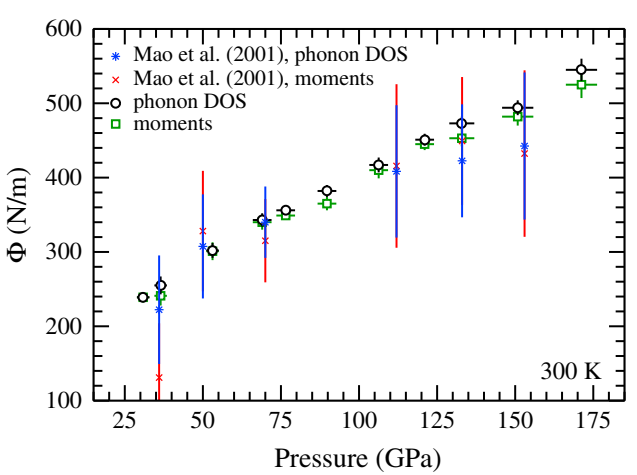

(b)

Figure 2. Average force constant of $\varepsilon$-Fe from NRIXS data. (a) Black circles give the volume dependence of the average force constant acting on the resonant isotope $(\Phi)$ determined from our integrated phonon DOS for $\varepsilon-\mathrm{Fe}$ (equation (2)); green squares show $\Phi$ determined from the third-order moment of $S(E)$. For comparison, red downward triangles show $\Phi(V)$ reported by Lin et al. [2010] using a similar moments analysis method. (b) Black circles and green squares give the same values for $\Phi$ presented in Figure 2a, now as a function of pressure, which is determined from our in situ XRD measurements and the Vinet EOS parameters reported by Dewaele et al. [2006]. For comparison, we also plot $\Phi(P)$ from our PHOENIX analysis of the NRIXS data set on $\varepsilon$-Fe measured by Mao et al. [2001]; blue stars give $\Phi$ from their phonon DOS, and red x's give $\Phi$ from the third-order moment of their $S(E)$.

1956a, 1956b; Murphy et al., 2011b], which we calibrated in $P T$ space with reported melting points for $\varepsilon-F e$ [Shen et al., 1998; Ma et al., 2004; Komabayashi and Fei, 2010]. In particular, the values of $f_{\mathrm{LM}}$ given in Table 1 are closely related to our reported Lamb-Mössbauer temperatures, which were derived from a high-temperature formulation for $\left\langle u^{2}>\right.$ [Murphy et al., 2011b].

\section{Average Force Constant of $\varepsilon-F e$}

[16] The average force constant $(\Phi)$ acting on the resonant isotope in a crystal lattice at $300 \mathrm{~K}$ can be obtained directly from its total phonon DOS via

$$
\Phi(V)=\frac{m *}{3 \hbar^{2}} \int E^{2} D(E, V) d E,
$$

where $m^{*}$ is the mass of the resonant nucleus $\left({ }^{57} \mathrm{Fe}\right), \hbar$ is the reduced Planck constant, and the phonon DOS has been normalized by $\int D(E) \mathrm{d} E=3$ (Table 1 ). In addition, $\Phi$ can be obtained from the third-order moment of $S(E)$, in a procedure similar to that described in section 3 . We plot $\Phi(V)$ in Figure 2, where one can see that the values determined using the moments analysis method are slightly lower and more scattered than those obtained from the phonon DOS (equation (2)), although they agree within uncertainty at all compression points. The systematic offset and increased scatter from the moments analysis may reflect the existence of minor multiphonon contributions beyond our scanned energy range. We note that a similar source of uncertainty is not present in the phonon DOS determination of $\Phi(V)$ because the phonon DOS is determined from single-phonon contributions only, and there have been no theoretical reports of singlephonon modes beyond our scanned energy ranges [e.g., Sha and Cohen, 2010]. Finally, a comparison of the moments analysis determinations of $f_{\mathrm{LM}}(V)$ and $\Phi(V)$ (Figures 1 and 2) reveals larger scatter in the latter parameter, which results from the fact that the average force constant arises from a higher-order moment; in particular, higher-order moments contribute more in the high-energy region of the measured NRIXS data where counting rates are inherently low and, in turn, statistical fluctuations result in larger uncertainties.

[17] To investigate the influence of data collection time and the energy range of NRIXS experiments on the determination of $\Phi(V)$ for $\varepsilon$-Fe, we present a comparison with the results of previous NRIXS experiments to similar pressures in Figures $2 \mathrm{a}$ and $2 \mathrm{~b}$. Figure $2 \mathrm{~b}$ demonstrates the importance of a high-statistical quality data set; although the present results agree within uncertainty with those determined from our PHOENIX analysis of the NRIXS data reported by Mao et al. [2001] (blue stars and red X's), it is obvious that longer data collection times result in significantly smaller uncertainties. Lin et al. [2010] also collected high-statistical quality data points at densities of 12.159 and $12.338 \mathrm{~g} / \mathrm{cm}^{3}$ $\left(V=4.684\right.$ and $4.616 \mathrm{~cm}^{3} / \mathrm{mol}$ accounting for isotopic enrichment, or $P=158$ and $172 \mathrm{GPa}$, respectively), with an energy range of $\pm 100 \mathrm{meV}$ around the nuclear transition energy of ${ }^{57} \mathrm{Fe}$ and approximately 20 scans per compression point. In particular, Lin et al. [2010] used a similar moments analysis method to derive $\Phi\left(4.684 \mathrm{~cm}^{3} / \mathrm{mol}\right)=511.7 \pm 5.6 \mathrm{~N} / \mathrm{m}$ and $\Phi\left(4.616 \mathrm{~cm}^{3} / \mathrm{mol}\right)=531.4 \pm 8.8 \mathrm{~N} / \mathrm{m}$ (red downward triangles in Figure 2a), which are in reasonable agreement with our moments analysis determinations of $\Phi(4.70 \pm 0.02$ $\left.\mathrm{cm}^{3} / \mathrm{mol}\right)=482 \pm 12 \mathrm{~N} / \mathrm{m}$ and $\Phi\left(4.58 \pm 0.02 \mathrm{~cm}^{3} / \mathrm{mol}\right)=525$ $\pm 18 \mathrm{~N} / \mathrm{m}$ at $300 \mathrm{~K}$ (Figure 2a). Such agreement suggests that phonon contributions beyond our scanned energy range $(-70$ to $+90 \mathrm{meV}$ ) are minor, and/or are largely captured by the data analysis procedures of the PHOENIX software [Sturhahn, 2000].

\subsection{Obtaining the $\beta$-Factors of $\varepsilon$-Fe From $\Phi(V, T)$}

[18] The volume dependence of $\Phi$ can be used to investigate $\varepsilon$-Fe's reduced isotopic partition function ratios ( $\beta$-factors), which provide information about isotope fractionation during equilibrium processes involving solid $\varepsilon$-Fe at elevated pressures and temperatures. In particular, the $\beta$-factor between 


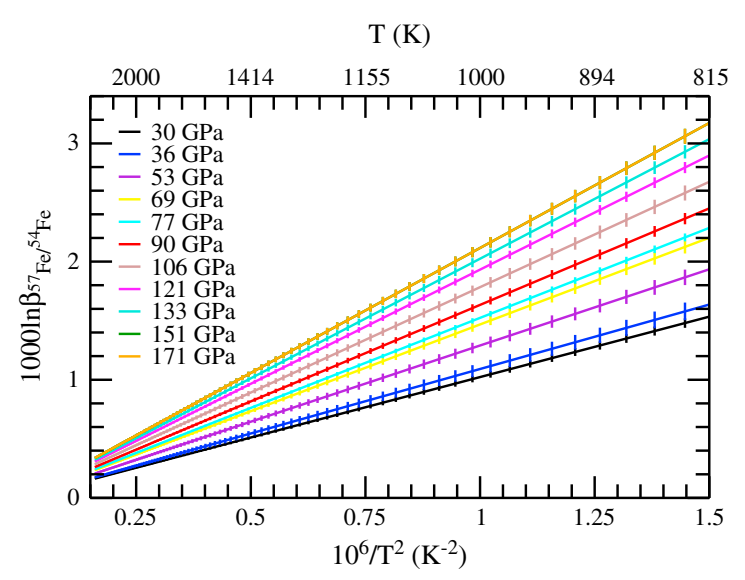

Figure 3. Reduced isotopic partition function ratios of $\varepsilon-\mathrm{Fe}$. Colored lines give the temperature dependence of the ${ }^{57} \mathrm{Fe} /{ }^{54} \mathrm{Fe}$ reduced isotopic partition function ratios $\left(1000 \ln \beta_{57 \mathrm{Fe} / 54 \mathrm{Fe}}\right)$ of $\varepsilon$-Fe between $T=815$ and $2500 \mathrm{~K}\left(10^{6} / T^{2}=1.5\right.$ and $0.16 \mathrm{~K}^{-2}$, respectively) using equation (8) and $\Phi(V)$ from the phonon DOS (equation (2)). Each color corresponds to an individual compression point, as labeled in the figure. Error bars reflect the propagation of measured uncertainties for $\Phi$ in equation (8), and are plotted at increments of $20 \mathrm{~K}$.

two isotopes at a given pressure is related to their free energies (F) via

$$
\ln \beta=-\frac{F^{*}-F}{k_{B} T}+\left(\frac{F^{*}-F}{k_{B} T}\right)_{\text {classical }},
$$

where $T$ is temperature, asterisks denote values for the isotopically substituted form, and the final subscript refers to values from classical mechanics [Bigeleisen and Mayer, 1947; Schauble, 2004]. From first-order thermodynamic perturbation theory, the difference between free energies of substituted and unsubstituted isotopic forms $\left(F^{*}-F\right)$ can be written in terms of the vibrational kinetic energy $\left(E_{\mathrm{K}}\right)$ and the difference in isotope masses,

$$
F^{*}-F=E_{K} \frac{\Delta m}{m}
$$

where $\Delta m=m-m^{*}$ (i.e., $\Delta m=-3$ when ${ }^{57} \mathrm{Fe}$ substitutes for ${ }^{54} \mathrm{Fe}$ ) [Polyakov and Mineev, 1999]. Together with the classical mechanics value of the kinetic energy, which is equal to $3 k_{\mathrm{B}} T / 2$, equations (3) and (4) can be combined to obtain

$$
\ln \beta=-\left(\frac{E_{K}}{k_{B} T}-\frac{3}{2}\right) \frac{\Delta m}{m}
$$

[19] In Murphy et al. [2011a], we reported values for the vibrational internal energy $\left(U_{\text {vib }}\right)$ from the integrated total phonon DOS, which comprises equal parts kinetic and potential energy components in the harmonic lattice approximation. Therefore, we can use our measured phonon DOS to determine $\ln \beta$ by applying $E_{\mathrm{K}}(V, T)=0.5 U_{\mathrm{vib}}(V, T)$ from Murphy et al. [2011a] to equation (5). However, accounting for measured uncertainties for $E_{\mathrm{K}}(V, T)$, we find that by the moderate temperature of $1000 \mathrm{~K}\left(10^{6} / T^{2}=1 \mathrm{~K}^{-2}\right)$, $\beta$-factors for our smallest and largest compression points are indistinguishable within uncertainty. In addition, by $\sim 1200 \mathrm{~K}$ $\left(\sim 0.7 \mathrm{~K}^{-2}\right)$, it becomes unclear whether the $\beta$-factors are positive or negative at all compression points, making it difficult to draw concrete conclusions about equilibrium isotope fractionation of $\varepsilon-\mathrm{Fe}$ at the temperatures that are relevant for Earth's mantle.

[20] Here we present an alternative approach to determining the pressure and temperature dependence of $\varepsilon$-Fe's $\beta$-factors from the phonon DOS, which reduces the uncertainties and, in turn, improves the available volume (pressure) and temperature resolution. If the temperature of interest is sufficiently high such that $k_{\mathrm{B}} T>>E_{\max }$, where $E_{\max }$ is the maximum (cut-off) energy of the phonon DOS, then we can approximate the vibrational kinetic energy by

$$
E_{K}(V, T)=\frac{3}{2} k_{B} T+\frac{1}{8 k_{B} T} \int E^{2} D(E, V, T) d E .
$$

[21] Based on inspection of our measured phonon DOS [Murphy et al., 2011a], we estimate that the vast majority of vibrational information lies at energies below $\sim 50$ and $75 \mathrm{meV}$ for our smallest and largest compression points, respectively, where the latter $E_{\max }$ implies that equation (6) is valid for $T>>800 \mathrm{~K}$.

[22] Substituting equation (6) into equation (5), we obtain

$$
\ln \beta(V, T)=-\frac{\Delta m}{m} \frac{1}{8\left(k_{B} T\right)^{2}} \int E^{2} D(E, V, T) d E
$$

whose integral is closely related to that given in the definition of $\Phi$ (equation (2)). Therefore, by combining equations (2) and (7), we obtain the expression

$$
\ln \beta(V, T)=-\frac{\Delta m}{m m^{*}} \frac{\hbar^{2} \Phi(V, T)}{8\left(k_{B} T\right)^{2}},
$$

which is valid above $800 \mathrm{~K}$ and based on $\Phi(V, T)$, where the temperature dependence of $\Phi$ is dictated by the quasiharmonic oscillator model. It has been suggested previously that anharmonic effects may be minor at the extreme compressions discussed here [Polyakov, 1998, 2009], but it is possible that the quasi-harmonic model does not accurately describe the behavior of $\varepsilon-\mathrm{Fe}$ at high temperatures. We rely on the quasi-harmonic model here because of a lack of sufficient data on the temperature dependence of $\varepsilon$-Fe's phonon DOS at large compressions.

[23] In Figure 3 , the ${ }^{57} \mathrm{Fe} /{ }^{54} \mathrm{Fe} \beta$-factors for $\varepsilon$-Fe determined using equation (8) and $\Phi(V)$ from the phonon DOS (equation (2)) at each of our compression points are plotted as separate lines as a function of inverse temperature $\left(10^{6} / T^{2}\right)$ between 815 and $2500 \mathrm{~K}$. Error bars are given at intervals of $20 \mathrm{~K}$, and correspond to the propagation of our measured uncertainties for $\Phi(V, T)$ in equation (8). The resulting average uncertainty for $\varepsilon$-Fe's $\beta$-factors is $\sim 2.5 \%$ over our experimental compression range, with a maximum uncertainty of $4.7 \%$ for $P=36 \pm 2 \mathrm{GPa}$, the compression point at which we collected the fewest NRIXS scans (section 2). For comparison, the errors produced when applying our $E_{\mathrm{K}}(V, T)$ to equation (5) are between 5 and $16 \%$ at $300 \mathrm{~K}$, and their relative magnitude increases with temperature. Finally, we note that our reported high-pressure $\beta$-factors for $\varepsilon$-Fe imply a fairly large pressure dependence, particularly at lower temperatures. Experiments directly probing the equilibrium isotopic partitioning of iron 


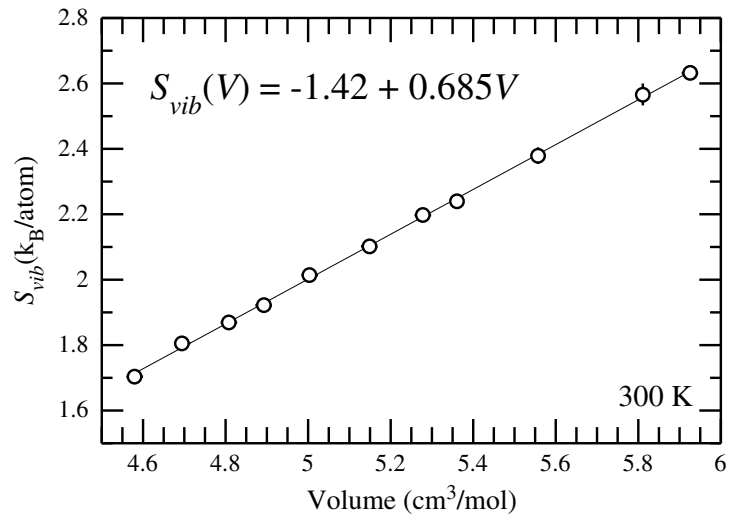

Figure 4. Vibrational entropy of $\varepsilon$-Fe. Black circles give the vibrational entropy $\left(S_{\mathrm{vib}}\right)$ at each compression point and $300 \mathrm{~K}$ (equation (9)); the black line gives the errors-weighted linear fit of our data, the result of which is given on the figure. We note that the slope reported here is equivalent to the value given in the text $(5.70 \pm 0.05 \mathrm{MPa} / \mathrm{K})$ via a conversion of units.

at high- $P T$ conditions are needed to confirm or refute this pressure dependence.

[24] Polyakov [2009] performed a similar analysis using equation (5) and $E_{\mathrm{K}}(V, T)$ from a previously published NRIXS data set up to $153 \mathrm{GPa}$ [Mao et al., 2001]. We note that the results from our analyses using both equations (5) and (8) agree fairly well with those reported by Polyakov [2009], but the results reported by Polyakov [2009] reflect the large scatter that is present in the data set measured by Mao et al. [2001]. In addition, we recall that the larger uncertainties from applying our $E_{\mathrm{K}}(V, T)$ to equation (5) produced indistinguishable $\beta$-factors over our entire compression range by a temperature of $1000 \mathrm{~K}$; by contrast, our analysis using equation (8) resolves a slight volume (pressure) dependence between most neighboring compression points up to at least $2000 \mathrm{~K}$. These differences cannot be resolved in the analysis presented by Polyakov [2009]. Therefore, we argue that obtaining $\beta$-factors via the average force constant is a superior approach for evaluating the high$P T$ partitioning behavior of iron isotopes in equilibrium processes involving solid $\varepsilon$-Fe.

[25] Dauphas et al. [2012] recently presented a similar method for obtaining $\beta$-factors from the average force constant, based on NRIXS experiments on iron-bearing oxides. However, their derivation involved $\Phi(V)$ from the moments analysis method, which led them to caution that average force constants and, in turn, $\beta$-factors obtained from NRIXS data collected over a limited energy range (i.e., less than $\pm 120 \mathrm{meV}$ ) might be inaccurate because higher-energy multiphonon contributions and/or normal vibrational modes could exist. We avoid this uncertainty by using $\Phi(V)$ from the phonon DOS (equation (2)) to determine $\varepsilon$-Fe's $\beta$-factors, because the phonon DOS is derived from singlephonon contributions only, and there have been no theoretical reports of single-phonon modes beyond our scanned energy ranges [e.g., Sha and Cohen, 2010]. However, for completeness, we now investigate the significance of potential missing multiphonon contributions by calculating $\beta$-factors for $\varepsilon$-Fe from our moments analysis determinations of $\Phi(V)$ at our two largest compression points, where vibrational information may extend to higher- energies. Using $\Phi(V)$ obtained from the third-order moment of our $S(E)$, we determine $10^{3} \ln \beta_{57 \mathrm{Fe} / 54 \mathrm{Fe}}\left(4.70 \pm 0.02 \mathrm{~cm}^{3} / \mathrm{mol}\right)=$ $22.9 \pm 0.6$ and $10^{3} \ln \beta_{57 \mathrm{Fe} / 54 \mathrm{Fe}}\left(4.58 \pm 0.02 \mathrm{~cm}^{3} / \mathrm{mol}\right)=25.0$ \pm 0.8 at $300 \mathrm{~K}$, which agree well with $\beta$-factors derived from our integrated phonon DOS (Table 1). Finally, we can also compare this result with the $\beta$-factors based on $\Phi(V)$ reported by Lin et al. [2010], the latter of which were determined from a moments analysis of NRIXS data collected over an energy range of $\pm 100 \mathrm{meV}$ (see Table 1 in reference). The average force constants measured by Lin et al. [2010] (section 4) provide $10^{3} \ln \beta_{57 \mathrm{Fe} / 34 \mathrm{Fe}}\left(4.684 \mathrm{~cm}^{3} / \mathrm{mol}\right)=24.3 \pm 0.3$ and $10^{3} \ln \beta_{57 \mathrm{Fe} / 54 \mathrm{Fe}}$ $\left(4.616 \mathrm{~cm}^{3} / \mathrm{mol}\right)=25.3 \pm 0.4$, which agree fairly well with the values given above, thus further supporting the claim that we capture most of the detail regarding multiphonon contributions over our scanned energy range.

\section{Entropy and Its Relation to the Thermal Expansion Coefficient of $\boldsymbol{\varepsilon}$-Fe}

[26] The vibrational entropy $\left(S_{\text {vib }}\right)$ per ${ }^{57} \mathrm{Fe}$ atom at $300 \mathrm{~K}$ can be obtained directly from the integrated phonon DOS via

$$
\begin{aligned}
S_{\mathrm{vib}}(V)= & \frac{1}{2 T} \int E \operatorname{coth} \frac{E}{2 k_{B} T} D(E, V) d E \\
& -k_{B} \int \ln \left(2 \sinh \frac{E}{2 k_{B} T}\right) D(E, V) d E,
\end{aligned}
$$

where the phonon DOS has been normalized by $\int D(E) \mathrm{d} E=3$ [Sturhahn, 2004]. Values for $S_{\text {vib }}(V)$ for $\varepsilon$-Fe determined from equation (9) are plotted in Figure 4, where one can see that $S_{\mathrm{vib}}$ has an approximately linear dependence, and that it decreases with decreasing volume (Table 1).

[27] The volumetric derivative of $S_{\text {vib }}$ at constant temperature is related to the vibrational thermal expansion coefficient $\left(\alpha_{\mathrm{vib}}\right)$ via thermodynamic definition,

$$
\left(\frac{\partial S_{\mathrm{vib}}}{\partial V}\right)_{\mathrm{T}}=\alpha_{\mathrm{vib}} K_{\mathrm{T}}
$$

where $K_{\mathrm{T}}$ is the isothermal bulk modulus. Because our $S_{\text {vib }}(V)$ is approximately linear, our results suggest that the product $\alpha_{\mathrm{vib}} K_{\mathrm{T}}$ is independent of volume at a constant temperature of $300 \mathrm{~K}$. Therefore, taking the derivative of an errors-weighted linear fit of our measured $S_{\mathrm{vib}}(V)$, we find $\left(\partial S_{\mathrm{vib}} / \partial V\right)_{300 \mathrm{~K}}=5.70 \pm 0.05 \mathrm{MPa} / \mathrm{K}$. We note that the slope given in Figure 4 of $0.685\left(k_{\mathrm{B}} / \mathrm{atom}\right) /\left(\mathrm{cm}^{3} / \mathrm{mol}\right)$ is equal to the slope given here via a conversion of units. For comparison, the corresponding electronic component for $\varepsilon$-Fe was calculated to be $\alpha_{\mathrm{el}} K_{\mathrm{T}} \sim 0.25 \mathrm{MPa} / \mathrm{K}$ [Wasserman et al., 1996], which is a factor of 20 smaller than our reported vibrational component at $300 \mathrm{~K}$.

[28] Applying our $\left(\partial S_{\mathrm{vib}} / \partial V_{300 \mathrm{~K}}\right.$ result and $K_{\mathrm{T}}(V, 300 \mathrm{~K})$ from the Vinet equation of state for $\varepsilon$-Fe reported by Dewaele et al. [2006], we find $\alpha_{\text {vib }}(300 \mathrm{~K})=1.84 \pm 0.0210^{-5} \mathrm{~K}^{-1}$ and $0.67 \pm 0.0210^{-5} \mathrm{~K}^{-1}$ at 30 and $171 \mathrm{GPa}$, respectively, where reported errors reflect the uncertainties associated with our fitting procedure (Table 1 and Figure 5). The variation in EOS parameters reported from XRD studies performed under varying degrees of hydrostaticity introduces a small additional uncertainty in $\alpha_{\text {vib }}$ for our largest compression points. For example, if we apply $K_{\mathrm{T}}(V)$ from the nonhydrostatic third-order BM EOS reported by Mao et al. [1990] to 


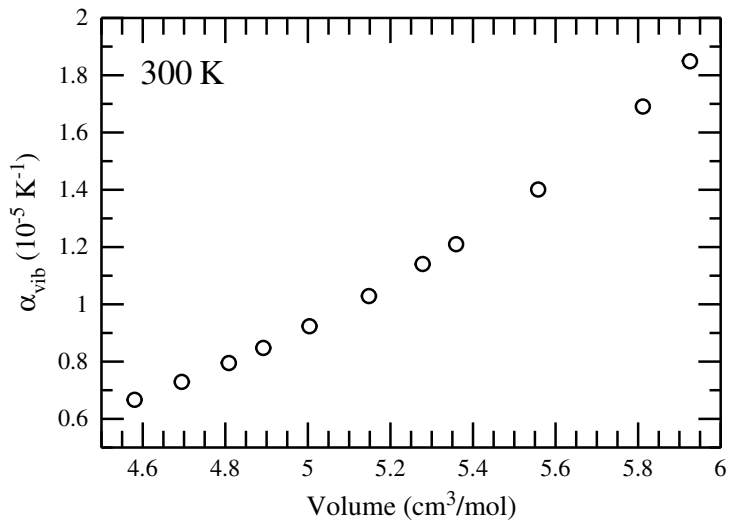

Figure 5. Vibrational thermal expansion coefficient of $\varepsilon-\mathrm{Fe}$ at $300 \mathrm{~K}$. The volume dependence of the vibrational thermal expansion coefficient $\left(\alpha_{\mathrm{vib}}\right)$ was determined using our measured $S_{\mathrm{vib}}(V)$, equation (10), and Vinet EOS parameters reported by Dewaele et al. [2006].

equation (10), we obtain $\alpha_{\text {vib }}(300 \mathrm{~K})=1.83 \pm 0.0210^{-5} \mathrm{~K}^{-1}$ and $0.61 \pm 0.0210^{-5} \mathrm{~K}^{-1}$ at 30 and $171 \mathrm{GPa}$, respectively, the latter of which is slightly beyond the mutual determined uncertainties.

[29] Our $\alpha_{\text {vib }}(V)$ for $\varepsilon$-Fe (Table 1) agrees well with results from first-principles calculations by Sha and Cohen [2010]; based on Figure 6 in the reference, we approximate their $\alpha_{\mathrm{vib}}(300 \mathrm{~K})=0.9 \times 10^{-5} \mathrm{~K}^{-1}$ and $0.6 \times 10^{-5} \mathrm{~K}^{-1}$ at 100 and $200 \mathrm{GPa}$, respectively. In addition, our $\alpha_{\mathrm{vib}}(V)$ agrees fairly well with the results of shock-compression experiments by Jeanloz [1979] at larger compression (see Figure 2 in reference). Based on the reported fitting equations for the bulk modulus and $\alpha$ along the Hugoniot $\left(K_{\mathrm{S}, \mathrm{H}}\right.$ and $\alpha_{\mathrm{H}}$, respectively), Jeanloz [1979] found $\alpha_{\mathrm{H}}(90 \mathrm{GPa})=1.2 \times 10^{-5} \mathrm{~K}^{-1}$ and $\alpha_{\mathrm{H}}(171 \mathrm{GPa})=0.7 \times 10^{-5} \mathrm{~K}^{-1}$. However, at smaller compressions, our values differ beyond uncertainties, with their reported $\alpha_{\mathrm{H}}(30 \mathrm{GPa})=2.6 \times 10^{-5} \mathrm{~K}^{-1}$. This large discrepancy at small compressions may be the result of different experimental conditions, i.e., shock-compression experiments are adiabatic, whereas our experiments are isothermal.

[30] It has been shown via theoretical calculations that the magnitude of the electronic thermal expansion coefficient increases with increasing temperature, while that of the vibrational component decreases [e.g., Wasserman et al., 1996]. This complicated combination of temperature, electronic, and vibrational effects are all included in $\alpha_{\mathrm{H}}$, whereas our experiments are isothermal and insensitive to the electronic contribution. Thus, the agreement between our results and those of Jeanloz [1979] suggests that the total thermal expansion coefficient is only weakly dependent on temperature, particularly at larger compressions. However, this argument is inconsistent with the conclusions of Alfè et al. [2001] and Wasserman et al. [1996], both of whom found $\alpha_{\mathrm{vib}} K_{\mathrm{T}}$ to have a significant temperature dependence. For example, Wasserman et al. [1996] report that at a pressure of $58 \mathrm{GPa}$, their $\alpha_{\mathrm{vib}} K_{\mathrm{T}}$ decreases by $\sim 10 \%$ between $T=1000$ and $6000 \mathrm{~K}$ due to anharmonic effects, but their overall $\alpha K_{\mathrm{T}}$ increases by $40 \%$ as a result of the rapidly increasing electronic contribution in metallic $\varepsilon$-Fe. We note that our $\alpha_{\text {vib }}(V, 300 \mathrm{~K})$ is indeed smaller than their plotted $\alpha_{\mathrm{vib}}(V)$ at elevated temperatures $(T \geq 1000 \mathrm{~K}$; see Figures 11 and 8 in references, respectively), but a quantitative comparison at $300 \mathrm{~K}$ is not straightforward from their figures alone.

[31] Finally, our $\alpha_{\mathrm{vib}}(V)$ is approximately half as large as $\alpha(V)$ reported by Anderson et al. [2001] and Isaak and Anderson [2003]. These two studies are related, and both are based on the differentiation of previously reported high- $P T$ XRD data collected for $\varepsilon$-Fe up to $305 \mathrm{GPa}$ and $1370 \mathrm{~K}$ [Dubrovinsky et al., 2000]. Comparing their reported values with our measurements at the most similar molar volumes per atom, Isaak and Anderson [2003] found $\alpha\left(5.9 \mathrm{~cm}^{3} / \mathrm{mol}\right)=3.88 \times 10^{-5} \mathrm{~K}^{-1}$ and $\alpha\left(4.9 \mathrm{~cm}^{3} / \mathrm{mol}\right)=$ $1.61 \times 10^{-5} \mathrm{~K}^{-1}$, both of which are roughly twice as large as our measured values (Table 1). We acknowledge that investigations of $\alpha$ from XRD include electronic contributions, to which our measurements are insensitive. However, based on the high-statistical quality of our data set and $\alpha_{\mathrm{e} 1} K_{\mathrm{T}} \sim 0.25 \mathrm{MPa} / \mathrm{K}$ at $300 \mathrm{~K}$ reported by Wasserman et al. [1996] (see Figure 8 in reference), we conclude that the our results do not agree with those of Anderson et al. [2001] and Isaak and Anderson [2003].

\subsection{Other Thermodynamic Parameters From $\alpha_{\text {vib }}$}

[32] Our knowledge of the product $\alpha_{\mathrm{vib}} K_{\mathrm{T}}$ allows us to investigate a number of other thermodynamic parameters for $\varepsilon$-Fe. For example, $\alpha_{\mathrm{vib}} K_{\mathrm{T}}$ is equal to the temperature derivative of the vibrational thermal pressure via thermodynamic definition and Maxwell's relations

$$
\alpha_{\mathrm{vib}} K_{T}=\left[\frac{1}{V}\left(\frac{\partial V}{\partial T}\right)_{\mathrm{P}}\right]\left[-V\left(\frac{\partial \mathrm{P}}{\partial V}\right)_{\mathrm{T}}\right]=\left(\frac{\partial P_{\mathrm{vib}}}{\partial T}\right)_{\mathrm{V}} .
$$

[33] In our previous study, we reported $\varepsilon$-Fe's harmonic vibrational thermal pressure $\left(P_{\text {vib }}^{\mathrm{h}}\right)$ to Earth's core conditions, based on the volume and temperature dependence of $\varepsilon$-Fe's vibrational free energy $\left(F_{\mathrm{vib}}\right)$ [Murphy et al., 2011b]. In particular, we obtained $F_{\text {vib }}$ from each of our measured phonon DOS, and determined $F_{\text {vib }}(V, 300 \mathrm{~K})$ from an errors-weighted quadratic fit. We then took the volume derivative of the resulting quadratic function and found $P_{\mathrm{vib}}^{\mathrm{h}}(V, 300 \mathrm{~K})=\left(\partial F_{\mathrm{vib}} / \partial V\right)_{300 \mathrm{~K}}=4.22-0.323 V$. To investigate $F_{\text {vib }}(V, T>300 \mathrm{~K})$ and, in turn, the temperature dependence of $P_{\text {vib }}^{\mathrm{h}}$, we applied the quasi-harmonic oscillator model to our measured phonon DOS and repeated the same procedure [Murphy et al., 2011b]. Then, by taking the temperature derivative of errors-weighted quadratic fits our $P_{\mathrm{vib}}^{\mathrm{h}}(V, T)$ at each compression point between $T=100$ and $1000 \mathrm{~K}$, we found $\alpha_{\mathrm{vib}} K_{\mathrm{T}}(300 \mathrm{~K})=5.5 \pm 0.2 \mathrm{MPa} / \mathrm{K}$. This result agrees well with the value determined here of $5.70 \pm 0.05 \mathrm{MPa} / \mathrm{K}$, and we attribute the $\sim 3 \%$ discrepancy between these two results to the fact that we are comparing derivatives of two parameters obtained from our experimental data.

[34] Based on the thermal pressure analysis presented in our previous study, we determined a core-density deficit (CDD) of 5.6 $\pm 0.2 \%$ [Murphy et al., 2011b], which implies that $\varepsilon-\mathrm{Fe}$ is significantly more dense than the solid inner core at the conditions of Earth's inner core boundary. A similar derivation is not reported here because we have restricted the present analysis to the temperature conditions of our experiments $(300 \mathrm{~K})$, and temperature effects - in addition to electronic effects - must be considered when estimating 


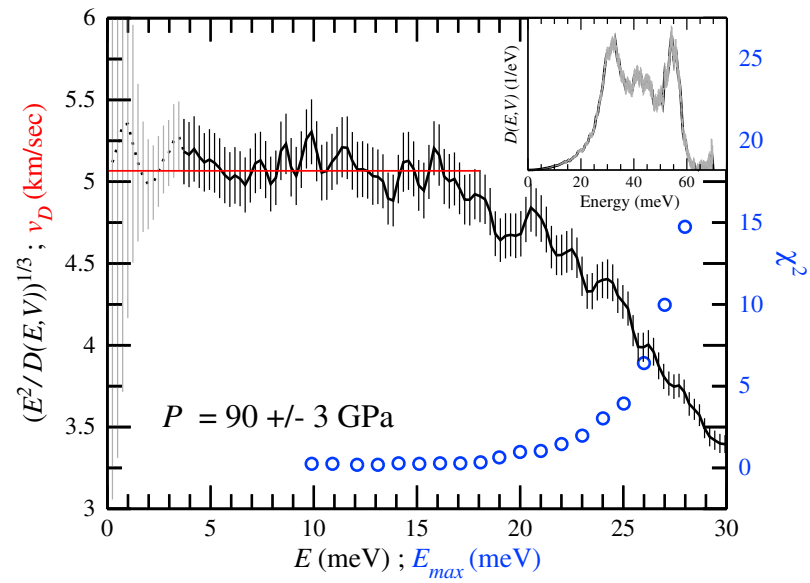

Figure 6. Representative determination of $v_{\mathrm{D}}$ from our NRIXS data. As an example, we show how the Debye sound velocity $\left(v_{\mathrm{D}}\right)$ was determined from the measured phonon DOS at $90 \pm 3 \mathrm{GPa}$, which is plotted with experimental error bars as a function of energy in the inset. The black curve in the main figure reflects the low-energy region of the phonon DOS, which has been inverted, scaled by energy-squared, and cube-rooted to allow for visual identification of the parabolic region as the energy-independent segment. The red line gives our fit of the parabolic region between 3.5 and $18 \mathrm{meV}$ and, in turn, $v_{\mathrm{D}}$, based on an exact relation for the dispersion of low-energy acoustic phonons and our determined densities. The minimum energy of the fitted region, demonstrated by the onset of the solid black line in the main figure, is slightly larger than the measured resolution function, and the maximum energy $\left(E_{\max }\right)$ was determined via a $\chi^{2}$ analysis of our parabolic fits, given by the blue circles.

the CDD. However, good agreement between our two analyses suggests that the presently derived thermal expansion coefficient of $\varepsilon-\mathrm{Fe}$ is consistent with our previous CDD. Such a result implies that a significant amount of light elements must be present in the solid inner core to match seismic observations, although the amount and identity of this light element component remains poorly constrained due to uncertainties in the stoichiometries, crystal structures, thermodynamics, and equations of state of alloys containing iron and candidate light elements for the core at high-PT conditions.

[35] Another parameter that can be determined from $\alpha_{\text {vib }} K_{\mathrm{T}}$ is the vibrational Grüneisen parameter, $\gamma_{\text {vib }}$, which is defined as

$$
\gamma_{\mathrm{vib}}=\frac{\alpha_{\mathrm{vib}} K_{\mathrm{T}} V}{C_{\mathrm{vib}}}
$$

[36] Thus, we can estimate $\gamma_{\text {vib }}(V)$ by applying our in situ measured sample volumes $(V)$, values for the vibrational specific heat capacity $\left(C_{\mathrm{vib}}\right)$ from the phonon DOS that were reported in our previous study [Murphy et al., 2011a], and $\left(\partial S_{\mathrm{vib}} / \partial V\right)_{300 \mathrm{~K}}$ from the present analysis to equation (12). This new analysis of $\gamma_{\text {vib }}$ agrees within uncertainty with our previous $\gamma_{\text {vib }}(V)$ - which was based on the volume dependence of the total phonon DOS-but equation (12) predicts a shallower slope than our original analysis presented in Murphy et al. [2011a]. Using the common parameterization $\gamma_{\text {vib }}(V)=\gamma_{\text {vib }, 0}\left(V / V_{0}\right)^{q}$, where the subscript " 0 " corresponds to ambient pressure conditions and $q$ determines the curvature of $\gamma_{\mathrm{vib}}(V)$, we previously reported a preferred $q$ range of 0.8 to 1.2 for $\gamma_{v i b, 0}=2.0 \pm 0.1$ [Murphy et al., 2011a]; the analysis using equation (12) predicts $q \sim 0.4$ and $\gamma_{\text {vib }, 0} \sim 1.6$. This apparent discrepancy highlights how difficult it is to constrain $q$ in the parameterization for $\gamma_{\text {vib }}$ written above, as noted in our previous study [Murphy et al., 2011a].

[37] The two methods for determining $\gamma_{\text {vib }}(V)$ agree fairly well at our larger compression points, with identical values at $V=4.89 \pm 0.02 \mathrm{~cm}^{3} / \mathrm{mol}$ and $4.81 \pm 0.02 \mathrm{~cm}^{3} / \mathrm{mol}$; at our largest compression point, $\gamma_{\mathrm{vib}}\left(4.58 \pm 0.02 \mathrm{~cm}^{3} / \mathrm{mol}\right)=1.40$ \pm 0.03 from equation (12), accounting for all reported uncertainties, and $1.34 \pm 0.1$ from our original analysis [Murphy et al., 2011a]. We note that the apparently larger uncertainty from our original analysis reflects the range of $q$ values included in the final reported fit to our individual $\gamma_{\text {vib }}$ data points. In fact, the fitting procedure described in Murphy et al. [2011a] for any of the individual $q$ values discussed (i.e., $q=0.8,1$, or 1.2) resulted in an uncertainty for $\gamma_{\text {vib }}$ of only 0.02 . We attribute the slightly larger uncertainty in the present analysis to the fact that the use of equation (12) involves a more circuitous path from the phonon DOS to $\gamma_{\mathrm{vib}}$, which relies on a number of independent parameters.

\section{Sound Velocities}

[38] A material's Debye sound velocity $\left(v_{\mathrm{D}}\right)$ is related to the low-energy region of its phonon DOS, which is parabolic ("Debye-like") [e.g., Sturhahn and Jackson, 2007]. We find that $\varepsilon$-Fe's phonon DOS has a substantial parabolic region (Figure 6), which allows us to determine $v_{\mathrm{D}}$ at each compression point by using an exact relation for the dispersion of low-energy acoustic phonons and our determined densities [Hu et al., 2003; Sturhahn and Jackson, 2007]. We note that the densities $(\rho)$ used in our sound velocity determinations are based on our in situ measured volumes and account for the isotopic enrichment of our samples, i.e., $m^{* 95 \%}=56.95 \mathrm{~g} / \mathrm{mol}$ for $95 \%$ isotopically enriched ${ }^{57} \mathrm{Fe}$ (Table 2 ).

[39] The energy ranges for the parabolic region from which we determine $v_{\mathrm{D}}$ at each compression point are given in Table 2. A typical minimum energy for the fit is $3.5 \mathrm{meV}$, which is slightly larger than the width of the measured resolution functions (section 2) [e.g., Murphy et al., 2011a]. The maximum energy for each compression point is determined using a $\chi^{2}$ analysis of our parabolic fits (Figure 6), and varies between 12 and $22 \mathrm{meV}$ (Table 2 ). In general, wider energy ranges correspond to larger compressions. However, smaller energy ranges are used for compression points where the maximum energy of the parabolic region is not clearly defined, i.e., where there is a gradual increase in $\chi^{2}$ with increasing width of the fitted region.

[40] The resulting $v_{\mathrm{D}}$ values are given in Table 2 and plotted in Figure 7, where one can see that there is no resolvable discontinuity in our measured sound velocities for NRIXS experiments performed in neon $(P \leq 69 \mathrm{GPa})$ and boron epoxy pressure transmitting media (Table 1). Typical uncertainties are $\leq 1 \%$, with the exception of our measurement at $\rho=12.13 \pm 0.03 \mathrm{~g} / \mathrm{cm}^{3}(P=151 \pm 5 \mathrm{GPa})$, which has a larger uncertainty because of a "tail" on the measured resolution function that extends to approximately $-20 \mathrm{meV}$. 
MURPHY ET AL.: THERMOELASTICITY OF hcp-Fe TO 171 GPa

Table 2. Elasticity of $\varepsilon$-Fe From the Phonon DOS

\begin{tabular}{|c|c|c|c|c|c|c|c|}
\hline$\rho\left(\mathrm{g} / \mathrm{cm}^{3}\right)^{\mathrm{a}}$ & $P(\mathrm{GPa})$ & Energy Range $(\mathrm{meV})^{\mathrm{b}}$ & $v_{\mathrm{D}}(\mathrm{km} / \mathrm{s})^{\mathrm{b}}$ & $K_{\mathrm{S}}(\mathrm{GPa})^{\mathrm{c}}$ & $v_{\mathrm{p}}(\mathrm{km} / \mathrm{s})^{\mathrm{c}}$ & $v_{\mathrm{s}}(\mathrm{km} / \mathrm{s})^{\mathrm{c}}$ & $\mu(\mathrm{GPa})^{\mathrm{c}}$ \\
\hline $9.61(3)$ & $30(2)$ & $3.5-15$ & $4.33(2)$ & 312 & $7.25(8)$ & $3.88(2)$ & $145(2)$ \\
\hline $9.80(1)$ & $36(2)$ & $3.5-12$ & $4.38(6)$ & 340 & $7.42(8)$ & $3.91(5)$ & $150(4)$ \\
\hline $10.25(1)$ & $53(2)$ & $3.5-18$ & 4.53(4) & 411 & 7.87(8) & 4.05(4) & $168(3)$ \\
\hline $10.63(1)$ & $69(3)$ & $3.5-16$ & $4.79(4)$ & 476 & $8.32(8)$ & $4.28(3)$ & $195(3)$ \\
\hline $10.80(2)$ & $77(3)$ & $3.5-18$ & $4.86(2)$ & 506 & $8.48(8)$ & $4.34(2)$ & 203(2) \\
\hline $11.06(2)$ & $90(3)$ & $3.5-18$ & $5.07(3)$ & 558 & $8.81(9)$ & $4.52(2)$ & $226(2)$ \\
\hline $11.38(5)$ & $106(3)$ & $3.5-18$ & $5.17(3)$ & 621 & $9.11(9)$ & 4.61(3) & $242(3)$ \\
\hline 11.64(2) & 121(3) & $3.5-15$ & $5.37(4)$ & 677 & $9.42(10)$ & 4.79(4) & 267(4) \\
\hline $11.84(2)$ & $133(4)$ & $3.5-17$ & $5.37(5)$ & 721 & $9.56(11)$ & $4.79(4)$ & $271(5)$ \\
\hline $12.13(3)$ & 151(5) & $9.7-18$ & $5.51(7)$ & 786 & $9.84(12)$ & $4.91(6)$ & $292(8)$ \\
\hline $12.43(3)$ & $171(5)$ & $3.7-22$ & $5.60(6)$ & 859 & $10.11(12)$ & $4.99(6)$ & $309(7)$ \\
\hline
\end{tabular}

${ }^{\mathrm{a}}$ Densities $(\rho)$ were determined from our in situ measured volumes (Table 1) and $m^{* 95 \%}=56.95 \mathrm{~g} / \mathrm{mol}$ for $95 \%$ isotopically enriched ${ }^{57} \mathrm{Fe}$.

${ }^{\mathrm{b}}$ The best energy range over which to fit the phonon DOS to obtain the Debye sound velocity $\left(v_{\mathrm{D}}\right)$ was determined by $\chi^{2}$ analysis (section 6$) ; v_{\mathrm{D}}$ at each compression point depends on our in situ measured volumes (densities) and accounts for ${ }^{57} \mathrm{Fe}$ enrichment levels.

${ }^{c}$ The adiabatic bulk modulus $\left(K_{\mathrm{S}}\right)$ was determined from the relationship $K_{\mathrm{S}}=K_{\mathrm{T}}(1+\alpha \gamma T)$, with the isothermal bulk modulus $\left(K_{\mathrm{T}}\right)$ reported by Dewaele et al. [2006], our $\alpha_{\text {vib }}$ from Table 1, and our previously reported $\gamma_{\text {vib }}$ [Murphy et al., 2011a]. $K_{\mathrm{S}}$ was used with our $\rho$ and $v_{\mathrm{D}}(\rho)$ to determine the compressional $\left(v_{\mathrm{p}}\right)$ and shear $\left(v_{\mathrm{s}}\right)$ sound velocities and the shear modulus $(\mu)$ for $\varepsilon$-Fe using equations (13) and (14). Reported uncertainties in the last significant digit(s) reflect uncertainties determined by the PHOENIX software [Sturhahn, 2000]. We note that uncertainties are not given for $K_{\mathrm{S}}$ because they would largely reflect uncertainties in the EOS parameters reported by Dewaele et al. [2006]; in particular, our uncertainties in $\alpha_{\text {vib }}$ and $\gamma_{\text {vib }}$ contribute a maximum error of only $0.2 \mathrm{GPa}$.

There is a slight difference in the values for $v_{\mathrm{D}}$ given in Table 2 and in Murphy et al. [2011a], which results from the use of a more restricted energy range in the present analysis. The Debye sound velocities reported here agree within uncertainties with those reported in Murphy et al. [2011a], except for our measurements at $77 \pm 3$ and $151 \pm 5 \mathrm{GPa}$. The disagreement at these two compression points likely reflects the fact that the cut-off energies for the parabolic region are not clearly defined. Finally, we note that when reperforming the analysis presented by Murphy et al. [2011a] for determining the Debye Grüneisen parameter from the density dependence of $v_{\mathrm{D}}$, the fitting parameters from the present analysis are identical to those reported in Murphy et al. [2011a].

[41] Here we use our measured $v_{\mathrm{D}}$ and $\rho$ to determine $\varepsilon$-Fe's compressional $\left(v_{\mathrm{p}}\right)$ and shear $\left(v_{\mathrm{s}}\right)$ sound velocities via

$$
\begin{gathered}
\frac{K_{\mathrm{S}}}{\rho}=v_{\mathrm{P}}^{2}-\frac{4}{3} v_{\mathrm{S}}^{2}, \\
\frac{3}{v_{\mathrm{D}}^{3}}=\frac{1}{v_{\mathrm{P}}^{3}}+\frac{2}{v_{\mathrm{S}}^{3}}
\end{gathered}
$$

[e.g., Sturhahn and Jackson, 2007], where the adiabatic bulk modulus $\left(K_{\mathrm{S}}\right)$ is related to the isothermal bulk modulus $\left(K_{\mathrm{T}}\right)$ via $K_{\mathrm{S}}=K_{\mathrm{T}}(1+\alpha \gamma T)$. To determine $K_{\mathrm{S}}$ at each compression point, we scale the ambient temperature $K_{\mathrm{T}}$ from the Vinet EOS reported by Dewaele et al. [2006] with the vibrational Grüneisen parameter $\left(\gamma_{\mathrm{vib}}\right)$ from Murphy et al. [2011a] and $\alpha_{\mathrm{vib}}$ from section 5 (Table 1). Using these parameters and accounting for uncertainties in $\alpha_{\text {vib }}$ and $\gamma_{\text {vib }}$, we find $\alpha_{\text {vib }} \gamma_{\mathrm{vib}} T<0.01$ over our compression range and at $300 \mathrm{~K}$, thus introducing a difference between $K_{\mathrm{S}}$ and $K_{\mathrm{T}}$ of less than $1 \%$ (Table 2). We note that electronic contributions to $\alpha$ and $\gamma$ are expected to be minor at $300 \mathrm{~K}$, based on the facts that $\alpha_{\mathrm{el}} / \alpha_{\mathrm{vib}} \approx 4 \%$ [Wasserman et al., 1996], and the influence of the electronic Grüneisen parameter (weighted by the electronic specific heat capacity) is thought to be negligible over this compression range [Boness et al., 1986; Alfè et al., 2001]. Finally, applying these values for $K_{\mathrm{S}}$ and our measured $v_{\mathrm{D}}$ and $\rho$ to equations (13) and (14), we determine $v_{\mathrm{p}}$ and $v_{\mathrm{s}}$ at each of our compression points. The results of this analysis are given in Table 2 and plotted in Figure 7 as a function of density. Discussion regarding the influence of EOS on the determination of $v_{\mathrm{p}}$ and $v_{\mathrm{s}}$ will be presented in the next section.

[42] An alternative method for determining $v_{\mathrm{s}}$ that does not rely on an existing EOS for $\varepsilon$-Fe is to apply our $v_{\mathrm{D}}$ to equation (14), along with previously reported values for $\varepsilon$-Fe's $v_{\mathrm{p}}$. For example, the high-energy resolution inelastic $\mathrm{X}$-ray scattering (HERIX) technique has been used to determine $\varepsilon$-Fe's compressional sound velocity by measuring the low-energy region of the dispersion curve for $\varepsilon$-Fe's longitudinal acoustic phonons (see section 6.1 for more details). Such experiments have been performed on polycrystalline $\varepsilon$-Fe to a pressure of 112 GPa [e.g., Fiquet et al., 2001; Antonangeli et al., 2004;

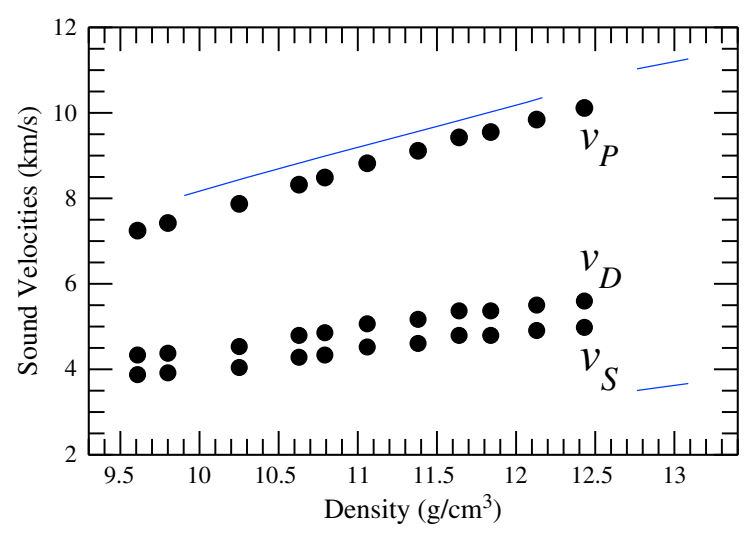

Figure 7. Our density-dependent sound velocities of $\varepsilon$-Fe at $300 \mathrm{~K}$ with PREM. Filled black circles give our preferred Debye $\left(v_{\mathrm{D}}\right)$, compressional $\left(v_{\mathrm{p}}\right)$, and shear $\left(v_{\mathrm{s}}\right)$ sound velocities for $\varepsilon$-Fe as a function of density, based on our NRIXS and in situ XRD experiments and the Vinet EOS measured by Dewaele et al. [2006] (Table 2). Uncertainties in sound velocities and densities are smaller than the symbol if not visible. Blue lines show $v_{\mathrm{p}}(\rho)$ and $v_{\mathrm{s}}(\rho)$ from PREM throughout Earth's core [Dziewonski and Anderson, 1981]; the discontinuity in $v_{\mathrm{p}}$ corresponds to the density jump across the ICB, and $v_{\mathrm{s}}=0$ in Earth's liquid outer core. 


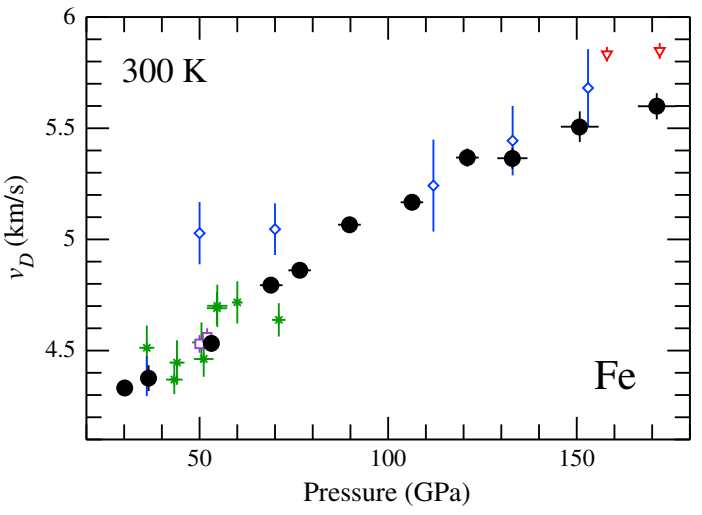

Figure 8. Debye sound velocities of $\varepsilon-F e$ at $300 \mathrm{~K}$. Filled black circles give our Debye sound velocities as a function of pressure, $v_{\mathrm{D}}(P)$, where our measured volumes have been converted to pressures using the Vinet EOS parameters reported by Dewaele et al. [2006] to facilitate comparison with previous studies (unfilled symbols). Also plotted are $v_{\mathrm{D}}(P)$ reported by Mao et al. [2001] (blue diamonds), Lin et al. [2005] (green X's), Mao et al. [2008] (purple squares), and Lin et al. [2010] (red downward triangles). We note that $v_{\mathrm{D}}(P)$ plotted from Lin et al. [2010] are taken from their experimental geometry that is most similar to ours, i.e., NRIXS measured along the compression axis (see Table 1 in reference).

Antonangeli et al., 2012; Mao et al., 2012]. Antonangeli et al. [2012] recently measured $v_{\mathrm{p}}(80 \mathrm{GPa})=8.83 \pm 0.14 \mathrm{~km} / \mathrm{s}$ and $v_{\mathrm{p}}(93 \mathrm{GPa})=9.04 \pm 0.21 \mathrm{~km} / \mathrm{s}$ at $300 \mathrm{~K}$, where their reported pressures are based on in situ XRD experiments and the thermal EOS for $\varepsilon$-Fe measured by Fiquet et al. [2007] (see Table 1 in reference). At the most similar compressions-based on sample volumes measured by in situ XRD in each study, but presented in terms of reported pressures for ease of comparison-our NRIXS experiments provide $\quad v_{\mathrm{D}}(77 \pm 3 \mathrm{GPa})=$ $4.86 \pm 0.02 \mathrm{~km} / \mathrm{s}$ and $v_{\mathrm{D}}(90 \pm 3 \mathrm{GPa})=5.07 \pm 0.03 \mathrm{~km} / \mathrm{s}$. Finally, applying these values to equation (14), we obtain $v_{s}=$ $4.33 \pm 0.03 \mathrm{~km} / \mathrm{s}$ and $4.52 \pm 0.03 \mathrm{~km} / \mathrm{s}$ at $\sim 77$ and $90 \mathrm{GPa}$, respectively, where the uncertainties reflect reported errors for both $v_{\mathrm{D}}$ and $v_{\mathrm{p}}$. These values for $v_{\mathrm{s}}$ are identical to those determined using equations (13) and (14), the Vinet EOS reported by Dewaele et al. [2006], and our determined thermoelastic parameters for $\varepsilon-\mathrm{Fe}$ (Table 2 ).

[43] To compare our results with one-dimensional seismic models, we include in Figure 7 the density-dependent sound velocities predicted by the preliminary reference Earth model (PREM) [Dziewonski and Anderson, 1981] for the liquid outer core $(P \sim 136$ to $329 \mathrm{GPa})$ and solid inner core ( 2329 to $364 \mathrm{GPa})$. The apparent discontinuity in PREM's $v_{\mathrm{p}}(\rho)$ corresponds to the density increase across the inner core boundary (ICB). From an errors-weighted least-squares linear fit of our measured $v_{\mathrm{p}}(\rho)$ (i.e., Birch's Law; [Birch, 1960, 1961]), we determine a slope of $1.04 \pm 0.02(\mathrm{~km} / \mathrm{s}) /\left(\mathrm{g} / \mathrm{cm}^{3}\right)$. Using this slope to extrapolate our $v_{\mathrm{p}}(\rho)$ to the expected density of $\varepsilon^{-57} \mathrm{Fe}$ at the pressure of the ICB $\left(14.3 \mathrm{~g} / \mathrm{cm}^{3}\right.$ from [Dewaele et al., 2006]), we find $v_{\mathrm{p}}(330 \mathrm{GPa}, 300 \mathrm{~K})$ for $\varepsilon-\mathrm{Fe}$ is $10.5 \%$ larger than the reported value from PREM on the inner core side of the ICB. Understanding the reasons for this offset is challenging, due to the fact that our experiments are performed at $300 \mathrm{~K}$ and the temperature at the ICB has an ill-constrained range of $\sim 5000$ to $7000 \mathrm{~K}$, based on $\varepsilon$-Fe's reported melting behavior [e.g., Sola and Alfè, 2009; Komabayashi and Fei, 2010; Jackson et al., 2013].

[44] Birch's law is an empirical relation based on the experimental determination of compressional sound velocities for a wide variety of Earth materials as a function of compression and, thus, it is only strictly relevant for $v_{\mathrm{p}}(\rho)$. However, in the absence of reliable information about the density dependence of $\varepsilon$-Fe's shear modulus beyond our compression range (Table 2), we use the same relationship to estimate $v_{\mathrm{s}}(330 \mathrm{GPa}, 300 \mathrm{~K})$. We find that the shear sound velocity of $\varepsilon-\mathrm{Fe}$ at $300 \mathrm{~K}$ is $68 \%$ larger than the reported value for PREM on the inner core side of the ICB.

\subsection{Comparison With Previous Studies of $\varepsilon$-Fe's Sound Velocities}

[45] To facilitate comparison with studies that did not measure sample volume with in situ XRD, we convert our measured volumes to pressures with the Vinet EOS for $\varepsilon$-Fe measured by Dewaele et al. [2006] (Table 2), using a natural iron sample. To the best of our knowledge, the resulting EOS is also appropriate for our ${ }^{57} \mathrm{Fe}$ isotopically enriched sample because the slight difference in mass compared to natural $\mathrm{Fe}(m=55.85 \mathrm{~g} / \mathrm{mol})$ should have a negligible effect on its elastic moduli (e.g., bulk modulus).

[46] The resulting $v_{\mathrm{D}}(P)$ are plotted in Figure 8, along with previously reported ambient temperature sound velocities for $\varepsilon$-Fe up to $172 \mathrm{GPa}$ [Mao et al., 2001; Lin et al., 2005; Mao et al., 2008; Lin et al., 2010]. Values from Lübbers et al. [2000] are not included because the energy scale used in that study was incorrect. In addition, we do not include reported values from Giefers et al. [2002] because their NRIXS experiments were performed on a purposefully textured sample, with the DAC oriented at an angle relative to the incident X-ray beam; without in situ XRD, it is difficult to know the true volume (pressure) of their measured data points. Finally, we note that a comparison of $\varepsilon$-Fe's shear sound velocities would look similar to Figure 8 because $v_{\mathrm{s}}$ and $v_{\mathrm{D}}$ are closely related, as can be seen in the numerator values of equation (14) and in Figure 7.

[47] In Figure 8, it is evident that our data provide a new tight constraint on the pressure dependence of $v_{\mathrm{D}}$ for $\varepsilon-\mathrm{Fe}$, particularly at smaller compressions where results from previous static-compression experiments are scattered. In addition, the high-statistical quality of our data produces errors that are $\sim 60 \%$ and $30 \%$ smaller than those measured by Mao et al. [2001] and Lin et al. [2005], respectively, based on similar PHOENIX analyses of their published NRIXS data sets. Finally, our $v_{\mathrm{D}}(150 \pm 5 \mathrm{GPa})$ is slightly lower than that measured by Mao et al. [2001] (blue diamonds) at a similar pressure of $153 \mathrm{GPa}$ (albeit within uncertainty), while $v_{\mathrm{D}}$ for our two largest compression points are significantly lower than those measured by Lin et al. [2010] using the same experimental geometry (Figure 8). It is unclear why our $v_{\mathrm{D}}$ differ by such a large amount from those measured by Lin et al. [2010] at similar densities (pressures), and further explanation is made difficult by the fact that Lin et al. [2010] present two large-compression data points independent of a systematic study with compression. When comparing values for $\Phi(V)$-which are based on the integrated phonon DOS - from the present study and from 


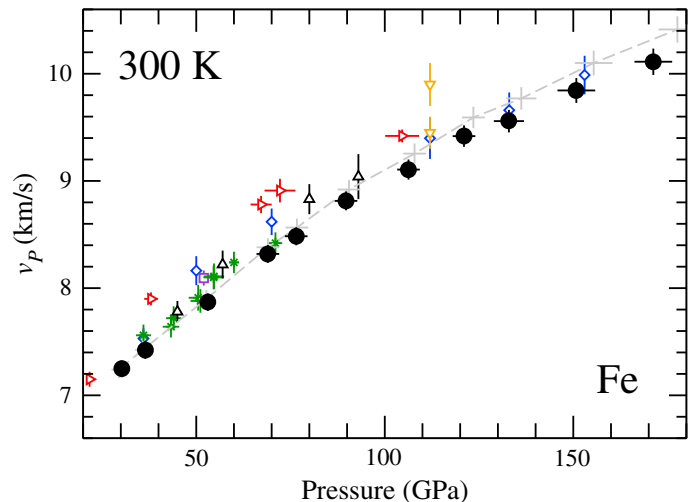

Figure 9. Compressional sound velocities of $\varepsilon$-Fe at $300 \mathrm{~K}$. Filled black circles give our compressional sound velocities as a function of pressure, $v_{\mathrm{p}}(P)$. To demonstrate the influence of the EOS used to extract $v_{\mathrm{p}}$ and $v_{\mathrm{s}}$, we include $v_{\mathrm{p}}(P)$ from our $v_{\mathrm{D}}(\rho)$ and the third-order BM EOS determined by Mao et al. [1990] (gray dashed line and crosses); we note that the different EOS produce a similar shift in pressure for $v_{\mathrm{s}}(P)$, but the values for $v_{\mathrm{s}}$ remain unchanged (section 6.1). Also plotted are $v_{\mathrm{p}}(P)$ from NRIXS experiments conducted by Mao et al. [2001] (blue diamonds), Lin et al. [2005] (green X's), and Mao et al. [2008] (purple square) on isotopically enriched iron; and $v_{\mathrm{p}}(P)$ from HERIX experiments on natural iron by Antonangeli et al. [2012] (black triangles), Mao et al. [2012] (red right triangles), and Antonangeli et al. [2004] (orange downward triangles).

Lin et al. [2010] (section 4), discrepancies remain but with smaller magnitudes; therefore, it is possible that such discrepant sound velocities result from different fitting procedures for determining $v_{\mathrm{D}}$ from the low-energy region of the phonon DOS (e.g., Figure 6 and section 6). Finally, we emphasize that this disagreement is unrelated to the fact that we apply the Vinet EOS from Dewaele et al. [2006] in our sound velocity determinations, while Lin et al. [2010] apply the third-order BM EOS from Mao et al. [1990], because $v_{\mathrm{D}}$ is derived from the low-energy region of the phonon DOS and sample density only. In fact, the disagreement between our $v_{\mathrm{D}}$ and those reported by Lin et al. [2010] becomes even larger if we apply the third-order BM EOS from Mao et al. [1990], because it shifts the pressure of our two largest compression points to 155 and $178 \mathrm{GPa}$, respectively.

[48] Next, we plot in Figure 9 the compressional sound velocities for $\varepsilon$-Fe as a function of pressure, where one can see that the overall trend of our $v_{\mathrm{p}}(P)$ (black filled circles) agrees fairly well with previous NRIXS studies on $\varepsilon$-Fe [Mao et al., 2001; Lin et al., 2005; Mao et al., 2008; Lin et al., 2010]. The better agreement among $v_{\mathrm{p}}(P)$ determined from NRIXS, as compared to $v_{\mathrm{D}}(P)$, is a result of the fact that $v_{\mathrm{p}}$ is strongly influenced by $K_{\mathrm{S}}$ (equation (13)), which is determined from existing EOS for $\varepsilon-F e$. To investigate the influence of the applied EOS on $v_{\mathrm{p}}(P)$, we include in Figure $9 v_{\mathrm{p}}(P)$ based on our NRIXS and in situ XRD data, with $K_{\mathrm{S}}$ from the third-order BM EOS reported by Mao et al. [1990] (gray dashed line and crosses), which was measured under nonhydrostatic conditions. The excellent agreement between $v_{\mathrm{p}}(P)$ determined from the two different
EOS for our four lowest-pressure points-where the sample was enclosed in a quasi-hydrostatic neon pressure mediumreflects the similarity of EOS measured under varying degrees of hydrostaticity at small compressions. At larger compressions, however, the sample environment under which the EOS was measured affects $K_{\mathrm{S}}(V)$ and, in turn, both the pressure and extracted $v_{\mathrm{p}}$ for a given compression point. In particular, $v_{\mathrm{p}}$ determined using the nonhydrostatic EOS reported by Mao et al. [1990] are slightly faster for our largest compression points, the pressures of which are also shifted to higher values (Figure 9). Finally, we note that these EOS produce values for $v_{\mathrm{s}}$ that are identical within uncertainty, because $v_{\mathrm{s}}$ is closely related to $v_{\mathrm{D}}$ (equation (14)) and independent of $K_{\mathrm{S}}$, by definition; however, the influence of the EOS on the pressure of a given compression point is the same as that depicted for $v_{\mathrm{p}}(P)$ in Figure 9.

[49] Another evident feature of Figure 9 is that the values and slopes of our $v_{\mathrm{p}}(P)$ agree fairly well with values reported in HERIX studies on $\varepsilon$-Fe performed by Antonangeli et al. [2004] and [2012] at $300 \mathrm{~K}$, while there is significant disagreement with those measured by Mao et al. [2012] using HERIX. One contributing factor to this disagreement is the fact that NRIXS experiments probe $95 \%$ isotopically enriched ${ }^{57} \mathrm{Fe}$ samples $\left(m^{* 95 \%}=56.95 \mathrm{~g} / \mathrm{mol}\right)$, while the HERIX experiments discussed here probed natural iron samples $(m=55.85 \mathrm{~g} / \mathrm{mol})$. In the harmonic oscillator model, a particle's vibrational frequency is inversely proportional to the square-root of its mass; thus, heavier isotopes oscillate with slower frequencies. In turn, following $v_{\mathrm{p}} \propto \mathrm{m}^{-1 / 2}$, one would expect the sound velocities of $\varepsilon-{ }^{57} \mathrm{Fe}$ probed with NRIXS to be $\sim 1 \%$ slower than those of natural $\varepsilon$-Fe probed with HERIX at a given volume. The density of $\varepsilon-{ }^{57} \mathrm{Fe}$ at a given volume is also $\sim 2 \%$ larger than that of natural $\varepsilon-\mathrm{Fe}$ and, therefore, the different isotopes probed in NRIXS and HERIX experiments explain part of the difference evident in Figure 9. However, isotopic mass alone cannot explain this discrepancy because the pressure derivatives of $v_{\mathrm{p}}(P)$ revealed by NRIXS and HERIX also appear to be different.

[50] Another likely explanation lies in the fact that NRIXS and HERIX access the compressional sound velocities of $\varepsilon$ Fe in slightly different ways. NRIXS probes the projected phonon DOS, which comprises nearly all lattice vibrations in the crystal based on the geometry and averaging procedures of the technique (see [Sturhahn and Jackson, 2007] for more details). The low-energy region of the phonon DOS - between 3.5 and 12 to $22 \mathrm{meV}$ in the present study (Table 2)-then, provides a Debye sound velocity that is averaged over essentially all crystallographic directions. In turn, the Debye sound velocity is used with an established EOS to determine the compressional sound velocity (section 6).

[51] HERIX measures points along the low-energy region of the dispersion curve, where the minimum accessible phonon energy is determined by the signal-to-noise ratio, energy resolution of the monochromator, selection rules, and separation of the diamond anvils' transverse acoustic peak from $\varepsilon$-Fe's longitudinal acoustic phonon peak. For example, Antonangeli et al. [2004] probed the phonon dispersion curve of $\varepsilon$-Fe for $E \geq 35 \mathrm{meV}$ (see Figure 4 in reference), while Mao et al. [2012] and Antonangeli et al. [2012] were able to measure $\varepsilon$-Fe's longitudinal acoustic mode for $E \geq 20 \mathrm{meV}$ (see Figure 1 in each reference). In turn, the authors determined $v_{\mathrm{p}}$ by taking the derivative of a sine-function fit of their 
measured acoustic phonon dispersion curves. Finally, HERIX's ability to select for longitudinal acoustic phonons allows for the investigation of longitudinal (compressional) sound velocities along specific crystallographic directions for single crystal samples. The same is approximately true for textured polycrystalline samples, assuming the preferred orientation directions are well known.

[52] To demonstrate the significance of this contrast, we highlight a comparison between our experimental conditions and results with those reported by Antonangeli et al. [2004] in their HERIX study. Antonangeli et al. [2004] prepared a DAC with purposefully nonhydrostatic sample conditions, to develop texture (preferred orientation) in their polycrystalline $\varepsilon$-Fe sample. They then investigated the phonon dispersion curve along directions that were $50^{\circ}$ and $90^{\circ}$ from the $c$-axis in $\varepsilon$-Fe by rotating the DAC with respect to the incident X-ray beam. In our experiments, we also observed some sample texturing in our five largest compression points $(P \geq 106 \mathrm{GPa})$, as indicated by a loss of intensity in the (002) diffraction peak (section 2). Although our sample texturing is similar to that presented by Antonangeli et al. [2004], we restate that our measured sound velocities are not similarly affected because they are obtained from the phonon DOS, which comprises phonons along nearly all lattice directions [Sturhahn, 2004].

[53] The different isotopic masses and directional sensitivities of our NRIXS experiments and the HERIX experiments performed by Antonangeli et al. [2004] make direct comparison challenging. One available method is to apply values for the elastic stiffness constants of $\varepsilon$-Fe to the Christoffel equation [Musgrave, 1970], in order to determine the sound velocities for all crystallographic directions. Then, by using the appropriate averaging procedures [Sturhahn, 2000; Sturhahn and Jackson, 2007], one can explore the relative directional sensitivity of sound velocities determined with NRIXS. The elastic stiffness constants of $\varepsilon$-Fe have not been measured because such a single-crystal does not yet exist with large enough dimensions, so we apply values from first-principles calculations at $52 \mathrm{GPa}$ and $300 \mathrm{~K}$ by Steinle-Neumann et al. [2004]. Using this procedure, $v_{\mathrm{p}}\left(0^{\circ}\right)$ is $\sim 1 \%(\sim 80 \mathrm{~m} / \mathrm{s})$ faster than $v_{\mathrm{p}}\left(90^{\circ}\right)$, where $0^{\circ}$ corresponds to a wave propagating along the $c$-axis direction. This result agrees qualitatively with the experiments by Antonangeli et al. [2004], who found that $v_{\mathrm{p}}\left(50^{\circ}\right)>v_{\mathrm{p}}\left(90^{\circ}\right)$ with a contrast of $450 \mathrm{~m} / \mathrm{s}$. However, the predicted magnitude of anisotropy from the Christoffel equation is smaller than that reported by Antonangeli et al. [2004], and in fact would not be detectable with NRIXS based on the uncertainties of our dataset (Table 2). In particular, if we apply the proper averaging procedures, then $v_{\mathrm{p}}(50 \mathrm{GPa})$ from NRIXS would be $\sim 10 \mathrm{~m} / \mathrm{s}$ faster than $v_{\mathrm{p}}\left(90^{\circ}\right)$, and $\sim 70 \mathrm{~m} / \mathrm{s}$ slower than $v_{\mathrm{p}}\left(0^{\circ}\right)$, both of which lie within our reported uncertainties at $\sim 50 \mathrm{GPa}$. We note that this argument is meant to be qualitative because there is a large amount of uncertainty associated with the elastic stiffness constants of $\varepsilon-\mathrm{Fe}$, which are the primary input parameters for this calculation.

\subsubsection{Alloying Effects}

[54] The Earth's core is thought to contain $\sim 5$ to $10 \mathrm{wt} \%$ nickel (Ni) and some light elements [McDonough, 2003], based on comparisons of seismic and cosmochemical observations with static- and shock-compression experiments on iron. Now that the compressional and shear sound velocities of pure iron have been firmly established to an outer core pressure of $171 \mathrm{GPa}$, an important next step is to investigate the effects of alloying light elements with iron on its thermoelastic properties.

[55] A significant theoretical and experimental effort has been dedicated to determining the sound velocities of iron alloyed with popular candidate light elements for the core (e.g., H, C, O, Si, and Si). In particular, HERIX [e.g., Badro et al., 2007; Antonangeli et al., 2010; Mao et al., 2012; Shibazaki et al., 2012] and NRIXS [e.g., Struzhkin et al., 2001; Lin et al., 2003b; Lin et al., 2004; Mao et al., 2004; Gao et al., 2009] have been used extensively to investigate the sound velocities of iron alloys. Although NRIXS is an isotope-selective technique, we note that it has been shown previously that the low-energy region of the phonon DOS provides the Debye sound velocity of the bulk sample [e.g., Hu et al., 2003]. Theoretical calculations [e.g., Stixrude et al., 1997; Steinle-Neumann et al., 2003; Vočadlo et al., 2009] and shock-compression experiments [e.g., Jeanloz, 1979; Brown and McQueen, 1986; Nguyen and Holmes, 2004; Huang et al., 2011] have also played an important role in this discussion, but we do not include their results here because they often report sound velocities at simultaneous high- $P T$ conditions.

[56] In Figure 10, we plot the pressure dependence of our measured Debye sound velocities ( $v_{\mathrm{D}}$; filled circles) with those reported from previous NRIXS studies on iron alloyed with H, C, Si, S, and $\mathrm{Ni}$ [Lin et al., 2003b; Lin et al., 2004; Mao et al., 2004; Gao et al., 2009]. One of the most striking features of Figure 10 is the limited pressure range over which iron alloys have been probed with NRIXS. A number of data points lie at pressures below the stability field of $\varepsilon-\mathrm{Fe}$ (i.e., $P<17.7 \mathrm{GPa}$ from [Dewaele et al., 2006]) and, thus, cannot be directly compared with our results. In subsequent figures, the maximum pressure plotted reflects the range over which our data overlap with existing data for the sound velocities of iron alloys, providing a better depiction of the relevant features that will be discussed in more detail.

[57] Finally, we note that although oxygen is a candidate light element for the Earth's core, we do not include FeO in our figures or discussion because of a current lack of sufficient sound velocity data. Struzhkin et al. [2001] investigated $\mathrm{FeO}$ in the diamond-anvil cell with NRIXS and reported a calculated curve for the sound velocities as a function of momentum transfer (see Figure $4 \mathrm{~b}$ in reference), not as a function of pressure. In addition, previous HERIX measurements of FeO by Badro et al. [2007] report $v_{\mathrm{p}}$ only as a function of density, without a clear explanation of the pressure range, crystal structure, or XRD measurements used to determine the amount of compression. As a result, a discussion of the effects of alloying oxygen with $\mathrm{Fe}$ is not straightforward and will not be included here.

\subsubsection{Alloying Effects on $\varepsilon$-Fe's Compressional Sound Velocities}

[58] We plot in Figure 11 the pressure dependence of our compressional sound velocities ( $v_{\mathrm{p}}$; filled circles) with those from NRIXS and HERIX studies of iron alloys up to $106 \mathrm{GPa}$. First considering only NRIXS results, it appears that the alloying of nickel and light elements does not significantly affect iron's compressional sound velocities at these pressures. In particular, distinctly different sound velocities for $\varepsilon$-Fe and iron alloys with systematic offsets are difficult to identify, especially for NRIXS experiments performed on $\mathrm{FeH}_{x}[$ Mao 


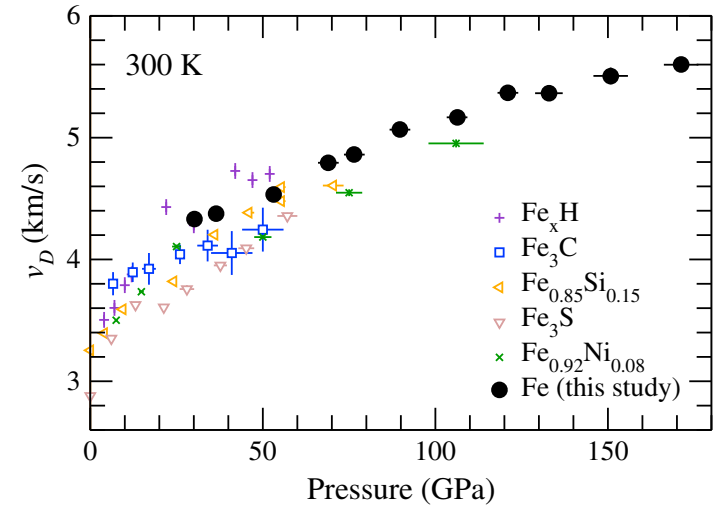

Figure 10. Debye sound velocities of $\varepsilon$-Fe and iron alloys. Filled black circles give our measured Debye sound velocities $\left(v_{\mathrm{D}}\right)$ for $\varepsilon-\mathrm{Fe}$ as a function of pressure. The remaining symbols give $v_{\mathrm{D}}(P)$ from NRIXS experiments on $\mathrm{FeH}_{x}$ (purple crosses [Mao et al., 2004]), $\mathrm{Fe}_{3} \mathrm{C}$ (blue squares [Gao et al., 2009]), $\mathrm{Fe}_{0.85} \mathrm{Si}_{0.15}$ (orange left triangles [Lin et al., 2003b]), $\mathrm{Fe}_{3} \mathrm{~S}$ (brown downward triangles [Lin et al., 2004]), and $\mathrm{Fe}_{0.92} \mathrm{Ni}_{0.08}$ (green x's [Lin et al., 2003b]).

et al., 2004] and $\mathrm{Fe}_{0.92} \mathrm{Ni}_{0.08}$ [Lin et al., 2003b]. It is possible that NRIXS experiments on $\mathrm{Fe}_{3} \mathrm{C}$ [Gao et al., 2009] and $\mathrm{Fe}_{0.85} \mathrm{Si}_{0.15}$ [Lin et al., 2003b] indicate slightly faster compressional sound velocities than those of $\varepsilon-\mathrm{Fe}$, while NRIXS experiments on $\mathrm{Fe}_{3} \mathrm{~S}$ [Lin et al., 2004] suggest slightly slower compressional sound velocities. However, these differences are largely within reported uncertainties, thus prohibiting further quantitative discussion.

[59] Also evident in Figure 11 is the seemingly systematic offset between NRIXS and HERIX results, where the latter tends to measure faster compressional sound velocities than the former for a given composition, possibly due, in part, to the isotopic enrichment of NRIXS samples (section 6.1). To facilitate comparisons among HERIX experiments and to avoid visual bias, we include in Figure $11 v_{\mathrm{p}}(P)$ for $\varepsilon$-Fe as measured by Antonangeli et al. [2012] (black triangles). A comparison of HERIX results for $\varepsilon$ - $\mathrm{Fe}$ and $\mathrm{Fe}_{0.78} \mathrm{Ni}_{0.22}$ [Kantor et al., 2007] indicate that 22 atomic percent Ni has only a slight effect on the compressional sound velocities of pure iron, although it stabilizes the face-centered cubic crystal structure over their experimental compression range $(12$ to $66 \mathrm{GPa})$ at $300 \mathrm{~K}$. By contrast, the compressional sound velocities measured by Lin et al. [2003b] for a lower-nickel content alloy $\left(\mathrm{Fe}_{0.92} \mathrm{Ni}_{0.08}\right)$ are attributed to the hcp unit cell, which is consistent with existing highpressure XRD studies of $\mathrm{Fe}_{0.9} \mathrm{Ni}_{0.1}$ [e.g., Tateno et al., 2012]. Despite their slightly different compositions and crystal structures, it is worth noting that $v_{\mathrm{p}}(P)$ measured by NRIXS and HERIX for these iron-nickel alloys agree well.

[60] Comparisons of HERIX results for $\varepsilon-\mathrm{Fe}$ with $\mathrm{Fe}_{3} \mathrm{C}$ [Fiquet et al., 2009], $\mathrm{Fe}_{0.85} \mathrm{Si}_{0.15}$ [Mao et al., 2012], and $\mathrm{Fe}_{0.89} \mathrm{Ni}_{0.04} \mathrm{Si}_{0.07}$ [Antonangeli et al., 2010] suggest that carbon and silicon increase the compressional sound velocities of Fe slightly, which is consistent with the conclusion drawn from NRIXS experiments. An increase is also seen in the compressional sound velocities of $\mathrm{FeH}_{\mathrm{x}}$ measured by HERIX [Shibazaki et al., 2012], but this trend is not observed in NRIXS experiments. One possible explanation for this discrepancy may lie in the unique challenge associated with determining the amount of hydrogen in the lattice of double hexagonal-close packed (dhcp) $\mathrm{FeH}_{x}$ [Mao et al., 2004; Shibazaki et al., 2012]. Hydrogen is difficult to detect with XRD, and its concentration cannot be measured in recovered samples because the hydrogen atoms escape the lattice upon decompression to ambient pressures. As a result, both Mao et al. [2004] and Shibazaki et al. [2012] estimate the composition of their dhcp- $\mathrm{FeH}_{\mathrm{x}}$ samples from its measured EOS [Hirao et al., 2004; Shibazaki et al., 2012], and determine $x \approx 1$. In turn, this inherent compositional uncertainty may play a role in the disagreement of their measured compressional sound velocities, where Mao et al. [2004] find $v_{\mathrm{p}}(P)$ for dhcp- $\mathrm{FeH}_{x}$ that are identical to our results for $\varepsilon$-Fe up to $52 \mathrm{GPa}$ using NRIXS, while Shibazaki et al. [2012] report $v_{\mathrm{p}}(P)$ from HERIX experiments up to $70 \mathrm{GPa}$ that are larger than those measured for $\varepsilon$-Fe with HERIX [Antonangeli et al., 2012] and have a different slope.

[61] The relatively large error bars on the reported compressional sound velocities of iron alloys - even at small compressions - make it difficult to further resolve and quantify the effects of alloying on $\varepsilon$-Fe's thermoelastic properties. In addition, the measurements for iron alloys discussed here were performed at pressures that are $\sim 1 / 2$ that of Earth's core-mantle boundary, and experimental uncertainties are likely to increase with compression as counting rates decrease and statistical fluctuations become more important. Some of

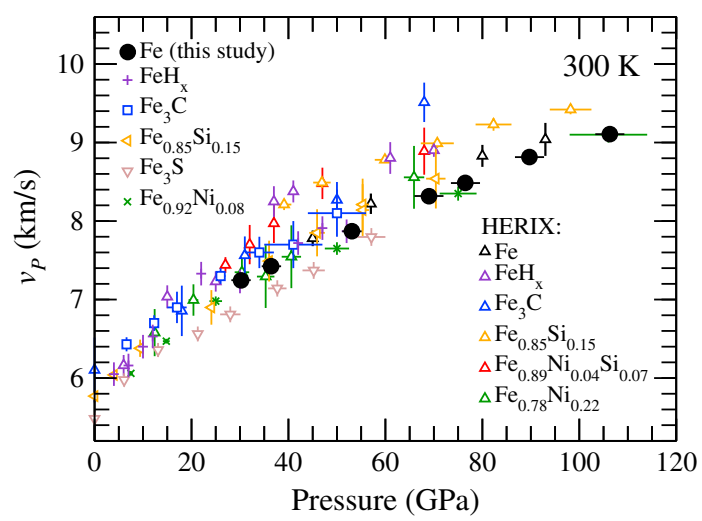

Figure 11. Compressional sound velocities of $\varepsilon$-Fe and iron alloys. Filled black circles give our measured compressional sound velocities $\left(v_{\mathrm{p}}\right)$ for $\varepsilon$-Fe as a function of pressure. We plot our $v_{\mathrm{p}}(P)$ only at pressures that overlap with reported values for iron alloys. $v_{\mathrm{p}}(P)$ from NRIXS experiments are plotted for isotopically enriched $\mathrm{FeH}_{x}$ (purple crosses [Mao et al., 2004]), $\mathrm{Fe}_{3} \mathrm{C}$ (blue squares [Gao et al., 2009]), $\mathrm{Fe}_{0.85} \mathrm{Si}_{0.15}$ (orange left triangles [Lin et al., 2003b]), $\mathrm{Fe}_{3} \mathrm{~S}$ (brown downward triangles [Lin et al., 2004]), and $\mathrm{Fe}_{0.92} \mathrm{Ni}_{0.08}$ (green x's [Lin et al., 2003b]). Unfilled triangles give $v_{\mathrm{p}}(P)$ from HERIX experiments on Fe (black [Antonangeli et al., 2012]), $\mathrm{FeH}_{x}$ (purple [Shibazaki et al., 2012]), $\mathrm{Fe}_{3} \mathrm{C}$ (blue [Fiquet et al., 2009]), $\mathrm{Fe}_{0.85} \mathrm{Si}_{0.15}$ (orange [Mao et al., 2012]), $\mathrm{Fe}_{0.89} \mathrm{Ni}_{0.04} \mathrm{Si}_{0.07}$ (red [Antonangeli et al., 2010]), and $\mathrm{Fe}_{0.78} \mathrm{Ni}_{0.22}$ (green [Kantor et al., 2007]) with natural isotopic abundances. 


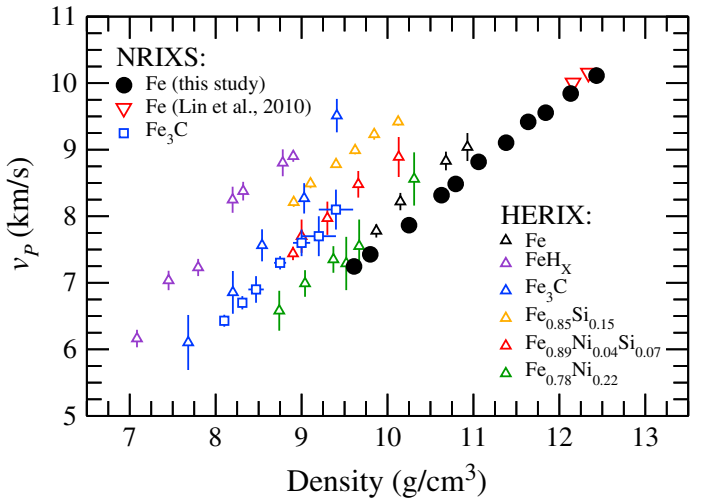

Figure 12. Density dependence of compressional sound velocities of $\varepsilon$-Fe and iron alloys. Filled black circles give our measured compressional sound velocities for $\varepsilon-F e$ as a function of density, $v_{\mathrm{p}}(\rho)$, the latter of which is determined from in situ XRD and $m^{* 95 \%}=56.95 \mathrm{~g} / \mathrm{mol}$ for $95 \%$ isotopically enriched ${ }^{57} \mathrm{Fe}$. Similarly, red downward triangles (blue squares) give $v_{\mathrm{p}}(\rho)$ for enriched $\varepsilon$ - $\mathrm{Fe}\left(\mathrm{Fe}_{3} \mathrm{C}\right)$ from NRIXS studies by Lin et al. [2010] (Gao et al. [2009]). Unfilled triangles give $v_{\mathrm{p}}(\rho)$ from HERIX experiments on iron and iron alloys with natural isotopic abundances $(m=55.85 \mathrm{~g} / \mathrm{mol}$ for iron): $\varepsilon$-Fe (black, [Antonangeli et al., 2012]), $\mathrm{FeH}_{x}$ (purple [Shibazaki et al., 2012]), $\mathrm{Fe}_{3} \mathrm{C}$ (blue [Fiquet et al., 2009]), $\mathrm{Fe}_{0.85} \mathrm{Si}_{0.15}$ (orange [Mao et al., 2012]), $\mathrm{Fe}_{0.89} \mathrm{Ni}_{0.04} \mathrm{Si}_{0.07}$ (red [Antonangeli et al., 2010]), and $\mathrm{Fe}_{0.78} \mathrm{Ni}_{0.22}$ (green [Kantor et al., 2007]).

these uncertainties can be reduced with the use of complementary in situ XRD experiments [e.g., Gao et al., 2009; Murphy et al., 2011a], which provide direct knowledge of the sample volume and, thus, density, resulting in increasingly accurate sound velocities (section 6). In addition, although an EOS is still needed if one uses equations (13) and (14) to obtain $v_{\mathrm{p}}$ and $v_{\mathrm{s}}$ from $v_{\mathrm{D}}$, we note that propagated uncertainties can be significantly reduced by eliminating the need for a secondary pressure scale, such as the pressure dependence of ruby fluorescence peaks.

[62] The iron-carbon system is one example where in situ XRD has been measured with both NRIXS and HERIX experiments, thus allowing for a more detailed comparison. In particular, Gao et al. [2009] and Fiquet et al. [2009] probed orthorhombic $\mathrm{Fe}_{3} \mathrm{C}$ with NRIXS and HERIX experiments up to 50 and $68 \mathrm{GPa}$, respectively, and they report sound velocities that agree within uncertainty up to $50 \mathrm{GPa}$ (Figure 11). However, Fiquet et al. [2009] measured a sharp increase in $v_{\mathrm{p}}$ at their largest compression point $(P=68 \mathrm{GPa})$, which is $\sim 12 \%$ larger than $v_{\mathrm{p}}(68 \mathrm{GPa})$ for $\varepsilon$-Fe, based on an interpolation between data points measured by Antonangeli et al. [2012] with HERIX. This effect can also be seen in a plot of $v_{\mathrm{p}}(\rho)$ (Figure 12), where we note that the relative magnitudes of the maximum densities of $\mathrm{Fe}_{3} \mathrm{C}$ differ from those of their maximum pressures because Gao et al. [2009] used an ${ }^{57} \mathrm{Fe}$-enriched sample. The underlying cause of this seemingly discontinuous increase in $v_{\mathrm{p}}$ reported by Fiquet et al. [2009] for $\mathrm{Fe}_{3} \mathrm{C}$ is not fully understood, but the authors note that they observed anomalous softening of the longitudinal acoustic branch at large momentum transfers above $68 \mathrm{GPa}$. The authors suggest that this vibrational instability may be related to a change in electronic state (e.g., a magnetic or high-spin to low-spin transition), which has been reported in theoretical and experimental studies as a potential cause for changes in the thermoelastic properties of iron-carbon alloys at a variety of pressures [e.g., Vočadlo et al., 2002; Lin et al., 2004; Chen et al., 2012; Prescher et al., 2012].

[63] Finally, we also plot in Figure 12 the density dependence of $v_{\mathrm{p}}$ from HERIX studies on $\varepsilon$-Fe and additional iron alloys [Kantor et al., 2007; Antonangeli et al., 2010, 2012; Mao et al., 2012; Shibazaki et al., 2012] that measured in situ XRD. The linear dependence of $v_{\mathrm{p}}$ on density at $300 \mathrm{~K}$ is consistent with the aforementioned Birch's Law for a wide range of iron alloys [Birch, 1960, 1961]. In addition, a comparison between $\varepsilon-\mathrm{Fe}$ [Antonangeli et al., 2012] and $\mathrm{Fe}_{0.78} \mathrm{Ni}_{0.22}$ [Kantor et al., 2007] measured with HERIX reveals similar absolute values and density derivatives, further supporting the claim that the alloying of nickel does not significantly influence the compressional sound velocities of pure iron.

6.1.3. Alloying Effects on $\varepsilon$-Fe's Shear Sound Velocities

[64] To begin our discussion of the effects of alloying on the shear sound velocities $\left(v_{\mathrm{s}}\right)$ of $\varepsilon-F e$, we recall that $v_{\mathrm{s}}$ for $\varepsilon$-Fe at $300 \mathrm{~K}$ is significantly larger than that predicted by PREM on the inner core side of the ICB (section 6) [Dziewonski and Anderson, 1981]. In Figure 13, we plot the pressure dependence of our determined shear sound velocities (filled circles) in the pressure range that overlaps

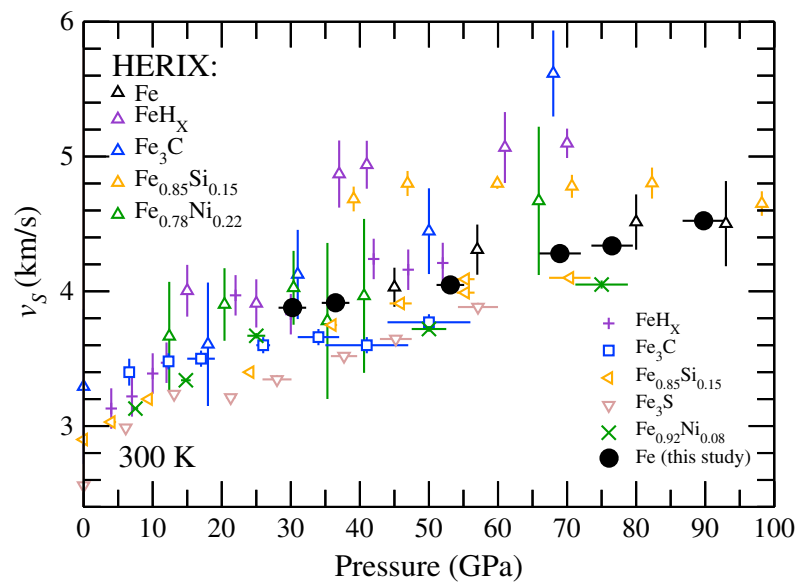

Figure 13. Shear sound velocities of $\varepsilon-\mathrm{Fe}$ and iron alloys. Filled black circles give our measured shear sound velocities $\left(v_{\mathrm{s}}\right)$ for $\varepsilon-\mathrm{Fe}$ as a function of pressure. We plot our $v_{\mathrm{s}}(P)$ only at pressures that overlap with reported values for iron alloys from NRIXS experiments on isotopically enriched $\mathrm{FeH}_{x}$ (purple crosses [Mao et al., 2004]), $\mathrm{Fe}_{3} \mathrm{C}$ (blue squares [Gao et al., 2009]), $\mathrm{Fe}_{0.85} \mathrm{Si}_{0.15}$ (orange left triangles [Lin et al., 2003b]), $\mathrm{Fe}_{3} \mathrm{~S}$ (brown downward triangles [Lin et al., 2004]), and $\mathrm{Fe}_{0.92} \mathrm{Ni}_{0.08}$ (green x's [Lin et al., 2003b]). Unfilled triangles give $v_{\mathrm{s}}(P)$ from HERIX experiments on iron and iron alloys with natural isotopic abundances, determined from their reported $v_{\mathrm{p}}(\rho)$ and existing EOS for each composition (section 6.1.3): $\varepsilon$-Fe (black, [Antonangeli et al., 2012]), $\mathrm{FeH}_{x}$ (purple [Shibazaki et al., 2012]), $\mathrm{Fe}_{3} \mathrm{C}$ (blue [Fiquet et al., 2009]), $\mathrm{Fe}_{0.85} \mathrm{Si}_{0.15}$ (orange [Mao et al., 2012]), and $\mathrm{Fe}_{0.78} \mathrm{Ni}_{0.22}$ (green [Kantor et al., 2007]). 
with those reported from previous NRIXS experiments [Lin et al., 2003b; Lin et al., 2004; Mao et al., 2004; Gao et al., 2009]. We reemphasize that the similar shapes of $v_{\mathrm{s}}(P)$ and $v_{\mathrm{D}}(P)$ (Figure 10) reflect the fact that NRIXS experiments are strongly sensitive to $v_{\mathrm{s}}$, thus placing a tighter constraint on $v_{\mathrm{s}}$ compared to $v_{\mathrm{p}}$.

[65] By contrast, it is very difficult to detect the shear modes (shear sound velocities) of iron alloys with HERIX experiments performed on polycrystalline samples, due to low signal to noise ratios and/or selection rules. In Figure 13, we plot $v_{\mathrm{s}}(P)$ from HERIX experiments on $\varepsilon$-Fe and select iron alloys whose EOS have been measured [Kantor et al., 2007; Fiquet et al., 2009; Antonangeli et al., 2012; Mao et al., 2012; Shibazaki et al., 2012], using a procedure similar to that described in section 6. In particular, we apply $v_{\mathrm{p}}(P)$ from HERIX experiments and $K_{\mathrm{T}}$ from their corresponding EOS [Mao et al., 1990; Scott et al., 2001; Lin et al., 2003a; Hirao et al., 2004] to equation (13) to determine $v_{\mathrm{s}}(P)$. Because of the previously discussed complications associated with comparing sound velocities determined from HERIX and NRIXS experiments, in addition to the fact that the described extraction of $v_{\mathrm{s}}(P)$ involves combining results from two independent experiments (per composition) in which the authors were not involved, the following discussion will not include a detailed comparison with $v_{\mathrm{s}}(P)$ determined from HERIX experiments on polycrystalline iron alloys. However, visual inspection of Figure 13 reveals that the relationship between $v_{\mathrm{s}}(P)$ determined from NRIXS and HERIX for a given composition is similar to the discussion presented in section 6.1.2 for $v_{\mathrm{p}}(P)$. In addition, the good agreement shown for pure $\varepsilon-\mathrm{Fe}$ is consistent with that found when determining $v_{\mathrm{s}}(P)$ for $\varepsilon$-Fe via equation (14), $v_{\mathrm{p}}$ from HERIX, and $v_{\mathrm{D}}$ from NRIXS in section 6 .

[66] It is apparent in Figure 13 that sound velocity experiments on iron alloys need to be performed over a wider pressure range in order to make reasonable inferences about their sound velocities at Earth's core conditions. A maximum of four data points for a given alloy overlap with our experimental compression range, and the largest compression point plotted in Figure 13 is at $70 \mathrm{GPa}$. We note that the shear sound velocities of hcp- $\mathrm{Fe}_{0.92} \mathrm{Ni}_{0.08}$ have been measured with NRIXS up to $106 \mathrm{GPa}$ [Lin et al., 2003b], and that they are $\sim 7 \%$ smaller than those of $\varepsilon-\mathrm{Fe}$, based on an average from the three overlapping compression points. In general, we conclude that while Ni may not have a strong influence on the density or compressional sound velocity of pure iron, its effect on the shear sound velocity could be significant.

[67] Another striking feature of Figure 13 is that the shear sound velocities of $\mathrm{dhcp}-\mathrm{FeH}_{x}$ are slightly larger than those of $\varepsilon$-Fe (albeit within the uncertainties). We note that this is opposite of the trend required for moving closer to seismic observations for Earth's inner core, independent of temperature effects. The addition of 15 atomic percent Si appears to slightly lower the shear sound velocities of $\varepsilon-\mathrm{Fe}$ [Lin et al., $2003 \mathrm{~b}$, although the authors observe no change in shear sound velocity between their two largest compression points, with $v_{\mathrm{s}}(55 \mathrm{GPa})=4.09 \pm 0.02 \mathrm{~km} / \mathrm{s}$ and $v_{\mathrm{s}}(70 \mathrm{GPa})=$ $4.10 \pm 0.02 \mathrm{~km} / \mathrm{s}$. Following $v_{\mathrm{s}}^{2}=\mu / \rho$, this constant value for $v_{\mathrm{s}}$ indicates that the increase in shear modulus $(\mu)$ of $\sim 6.7 \pm 2.4 \mathrm{GPa}(4.5 \%)$ between pressures of 55 and $70 \mathrm{GPa}$ arises entirely from the increase in density, given by the EOS for $\mathrm{Fe}_{0.85} \mathrm{Si}_{0.15}$ reported by Lin et al. [2003a] and accounting for isotopic enrichment. For comparison, the increase in $\mu$ between pressures of 46 and $55 \mathrm{GPa}$ is $12 \%$ $(17 \mathrm{GPa})$, indicating a different trend immediately before their largest compression point. Therefore, additional measurements beyond $70 \mathrm{GPa}$ are needed to clarify the trend for $v_{\mathrm{s}}(P)$ at larger compressions.

[68] A similar discussion applies to reported values for the shear sound velocities of orthorhombic $\mathrm{Fe}_{3} \mathrm{C}$, which were measured by Gao et al. [2009] up to $50 \mathrm{GPa}$ (Figure 13). Gao et al. [2009] report that their determined sound velocities at pressures above the magnetic collapse between 4.3 and $6.5 \mathrm{GPa}$ increase linearly with density, i.e., they do not report a softening in $v_{s}$. In particular, they find a slope of $v_{\mathrm{s}}(\rho)$ to be $\sim 0.24(\mathrm{~km} / \mathrm{s}) /\left(\mathrm{g} / \mathrm{cm}^{3}\right)$, compared to our reported value for $\varepsilon-F e$ of $0.42 \pm 0.02(\mathrm{~km} / \mathrm{s}) /\left(\mathrm{g} / \mathrm{cm}^{3}\right)$. It is possible that the small dip in shear sound velocity at $41 \mathrm{GPa}$ corresponds to a softening in $v_{\mathrm{s}}$ at that pressure, but with only a single larger compression point, it is difficult to determine whether a new trend in $v_{\mathrm{s}}(P)$ is being defined for $\mathrm{Fe}_{3} \mathrm{C}$ above this pressure, or perhaps that its slope is shallower than that of $\varepsilon$-Fe.

[69] Finally, Figure 13 shows a trend of $v_{\mathrm{s}}(P)$ for tetragonal $\mathrm{Fe}_{3} \mathrm{~S}$ that is distinctly lower than that of $\varepsilon-\mathrm{Fe}$, but with a similar slope. In particular, shear sound velocities for $\mathrm{Fe}_{3} \mathrm{~S}$ reported by Lin et al. [2004] are $\sim 8 \%$ smaller on average than those of $\varepsilon$-Fe at similar pressures. A larger compression range would be useful for determining an accurate slope of $v_{\mathrm{s}}(P)$ for $\mathrm{Fe}_{3} \mathrm{~S}$ and, in turn, whether $v_{\mathrm{s}}(P)$ for $\mathrm{Fe}_{3} \mathrm{~S}$ and $\varepsilon$-Fe remain subparallel or cross at a higher pressure.

\section{Concluding Remarks}

[70] We have determined a variety of thermoelastic and vibrational thermodynamic parameters for $\varepsilon$-Fe from the volume dependence of its total phonon DOS, which we measured with NRIXS and in situ XRD experiments between 30 and $171 \mathrm{GPa}$. From the integrated total phonon DOS, we found that the thermal atomic motion of $\varepsilon-\mathrm{Fe}$ is significantly reduced at outer-core pressures, as indicated by the steady increase in the Lamb-Mössbauer factor with compression. In addition, we determined $\varepsilon$-Fe's pressure- and temperaturedependent reduced isotopic partition function ratios ( $\beta$-factors) from its average force constant. Our technique produced smaller uncertainties and improved volume (pressure) resolution compared to methods involving the vibrational kinetic energy measured by NRIXS, thus allowing for more detailed analyses of the partitioning behavior of iron isotopes in equilibrium processes involving solid $\varepsilon$-Fe. Finally, we obtained $S_{\text {vib }}(V)$ from the integrated total phonon DOS, and used its volume derivative to determine the product of $\varepsilon$-Fe's vibrational thermal expansion coefficient and isothermal bulk modulus at $300 \mathrm{~K}$. In turn, $\left(\partial S_{\mathrm{vib}} / \partial V\right)_{300 \mathrm{~K}}$ allowed us to evaluate the temperature derivative of the harmonic thermal pressure and the volume dependence of the vibrational Grüneisen parameter. We found reasonable agreement between the present results and those reported in our previous studies, the latter of which were based on more direct determinations via the volume dependence of $\varepsilon$-Fe's total phonon DOS [Murphy et al., 2011b, 2011a]. 
[71] From the low-energy region of the phonon DOS, we obtained the Debye sound velocity of $\varepsilon$-Fe using a parabolic fit and, in turn, extracted its compressional and shear sound velocities via our determined densities, $\alpha_{\text {vib }}$, and $\gamma_{\text {vib }}$ for $\varepsilon$-Fe. Comparisons with compressional sound velocities measured by HERIX suggest an apparent systematic offset, with HERIX sound velocities being slightly faster than those determined by NRIXS. However, such comparisons are complicated, in part because NRIXS and HERIX access compressional sound velocities in slightly different ways. In addition, sound velocities for $\varepsilon$-Fe and iron alloys reported from NRIXS experiments are based on isotopically enriched samples, which should have slightly smaller vibrational frequencies at a given pressure and, in turn, slower sound velocities than samples from HERIX experiments that have natural isotopic abundances. The influence of this isotopic enrichment has yet to be fully quantified with experiments.

[72] We have also evaluated the effects of alloying on iron's compressional and shear sound velocities by comparing our determined values with those reported for iron alloys containing $\mathrm{Ni}$ and many candidate light elements for the core (e.g., H, C, Si, S). Considering only sound velocities determined from NRIXS experiments, we find that alloying does not significantly affect the compressional sound velocities of iron beyond reported uncertainties. In addition, we find that the alloying of $\mathrm{Ni}, \mathrm{C}, \mathrm{Si}$, or $\mathrm{S}$ results in slightly slower shear sound velocities than those measured for $\varepsilon$-Fe at the same pressure, while the alloying of $\mathrm{H}$ results in slightly faster shear sound velocities.

[73] It should be possible to combine our densities and sound velocities for $\varepsilon-\mathrm{Fe}$ with those reported for iron alloys, and invert the resulting data set to better constrain the composition of Earth's core via comparison with seismic observations. However, significant obstacles prevent such an analysis at this time, such as the limited compression range and large uncertainties associated with sound velocity measurements for iron alloys. In addition, although $\varepsilon$-Fe is thought to be the stable crystal structure of pure iron at the pressure and temperature conditions of Earth's core, the high- $P T$ stoichiometries and crystal structures of many candidate alloys have not been firmly established. Therefore, higher-statistical quality, larger compression ranges, and known stable crystal structures and stoichiometries for iron alloys - combined with the effects of temperature on the properties of $\varepsilon-\mathrm{Fe}$ and iron alloys - are necessary before such an inversion will be feasible.

\section{Notation}

$\begin{aligned} D(E, V) & \text { phonon density of states, } 1 / \mathrm{eV} \\ E & \text { energy, meV } \\ V & \text { molar volume per atom, } \mathrm{cm}^{3} / \mathrm{mol} \\ v_{\mathrm{D}} & \text { Debye sound velocity, } \mathrm{km} / \mathrm{s} \\ v_{\mathrm{p}} & \text { compressional sound velocity, } \mathrm{km} / \mathrm{s} \\ v_{\mathrm{s}} & \text { shear sound velocity, } \mathrm{km} / \mathrm{s} \\ f_{\mathrm{LM}} & \text { Lamb-Mössbauer factor, dimensionless } \\ E_{\mathrm{K}} & \text { vibrational kinetic energy, meV/atom } \\ \Phi & \text { average force constant, N/m } \\ \beta & \text { equilibrium isotopic partition function ratio (beta- } \\ & \text { factor), dimensionless }\end{aligned}$

$S_{\text {vib }} \quad$ vibrational entropy, $\mathrm{meV} / \mathrm{K} /$ atom

$\alpha_{\mathrm{vib}}$ vibrational thermal expansion coefficient, $\mathrm{K}^{-1}$

$C_{\text {vib }} \quad$ vibrational specific heat capacity, $\mathrm{meV} / \mathrm{K} /$ atom

$\gamma_{\text {vib }}$ vibrational Grüneisen parameter, dimensionless

$U_{\text {vib }}$ vibrational internal energy, meV/atom

$K_{\mathrm{T}}$ isothermal bulk modulus, $\mathrm{GPa}$

$K_{\mathrm{S}}$ adiabatic bulk modulus, $\mathrm{GPa}$

[74] Acknowledgments. We would like to thank B. Chen, D. Zhang, H. Yavas, and J.K. Wicks for assistance during the experiments, and NSFCAREER-0956166 and Caltech for support of this research. We would also like to thank two anonymous reviewers and the editor, Michael Walter, for helpful comments and suggestions. Use of the Advanced Photon Source was supported by the U.S. D.O.E., O.S., O.B.E.S. (DE-AC02-06CH11357). Sector 3 operations and the GSE-CARS gas-loading facility are supported in part by COMPRES (NSF EAR 06-49658).

\section{References}

Alfè, D., G. D. Price, and M. J. Gillan (2001), Thermodynamics of hexagonal-close-packed iron under Earth's core conditions, Phys. Rev. $B, 64(4), 16$, doi:10.1103/PhysRevB.64.045123.

Anderson, O. L., L. Dubrovinsky, S. K. Saxena, and T. LeBihan (2001), Experimental vibrational Grüneisen ratio values for epsilon-iron up to $330 \mathrm{GPa}$ at $300 \mathrm{~K}$, Geophys. Res. Lett., 28(12), 2359-2359, doi:10.1029/ $2001 \mathrm{GL} 013085$.

Antonangeli, D., F. Occelli, H. Requardt, J. Badro, G. Fiquet, and M. Krisch (2004), Elastic anisotropy in textured hep-iron to $112 \mathrm{GPa}$ from sound wave propagation measurements, Earth Planet. Sci. Lett., 225(1-2), 243-251, doi:10.1016/j.epsl.2004.06.004.

Antonangeli, D., J. Siebert, J. Badro, D. L. Farber, G. Fiquet, G. Morard, and F. J. Ryerson (2010), Composition of the Earth's inner core from high-pressure sound velocity measurements in Fe-Ni-Si alloys, Earth Planet. Sci. Lett., 295(1-2), 292-296, doi:10.1016/j.eps1.2010.04.018.

Antonangeli, D., T. Komabayashi, F. Occelli, E. Borissenko, A. C. Walters, G. Fiquet, and Y. Fei (2012), Simultaneous sound velocity and density measurements of hep iron up to $93 \mathrm{GPa}$ and $1100 \mathrm{~K}$ : An experimental test of the Birch's law at high temperature, Earth Planet. Sci. Lett., 331-332, 210-214, doi:10.1016/j.eps1.2012.03.024.

Badro, J., G. Fiquet, F. Guyot, E. Gregoryanz, F. Occelli, D. Antonangeli, and M. d'Astuto (2007), Effect of light elements on the sound velocities in solid iron: Implications for the composition of Earth's core, Earth Planet. Sci. Lett., 254(1-2), 233-238, doi:10.1016/j.epsl.2006.11.025.

Bigeleisen, J., and M. G. Mayer (1947), Calculation of equilibrium constants for isotopic exchange reactions, J. Chem. Phys., 15(5), 261-267, doi:10.1063/1.1746492.

Birch, F. (1960), The velocity of compressional waves in rocks to 10 kilobars, part 1, J. Geophys. Res., 65(4), 1083-1102, doi:10.1029/ JZ065i004p01083.

Birch, F. (1961), Velocity of compressional waves in rocks to 10 kilobars, part 2, J. Geophys. Res., 66(7), 2199-2224, doi:10.1029/ JZ066i007p02199.

Boehler, R., N. Vonbargen, and A. Chopelas (1990), Melting, Thermal Expansion, and Phase Transitions of Iron at High Pressures, J. Geophys. Res.-Solid Earth Planets, 95(B13), 21731-21736, doi:10.1029/ JB095iB13p21731.

Boness, D. A., J. M. Brown, and A. K. McMahan (1986), The Electronic Thermodynamics of Iron under Earth Core Conditions, Phys. Earth Planet. Inter., 42(4), 227-240.

Brown, J. M., and R. G. McQueen (1986), Phase Transitions, Grüneisen Parameter, and Elasticity for Shocked Iron Between $77 \mathrm{GPa}$ and $400 \mathrm{GPa}$, J. Geophys. Res.-Solid Earth Planets, 91(B7), 7485-7494, doi:10.1029/ JB091iB07p07485.

Cao, A. M., and B. Romanowicz (2004), Constraints on density and shear velocity contrasts at the inner core boundary, Geophys. J. Int., 157(3), 1146-1151, doi:10.1111/j.1365-246X.2004.02330.X.

Chen, B., L. L. Gao, B. Lavina, P. Dera, E. E. Alp, J. Y. Zhao, and J. Li (2012), Magneto-elastic coupling in compressed $\mathrm{Fe} 7 \mathrm{C} 3$ supports carbon in Earth's inner core, Geophys. Res. Lett., 39, L18301, doi:10.1029/ $2012 \mathrm{~g} 1052875$.

Dauphas, N., M. Roskosz, E. E. Alp, D. C. Golden, C. K. Sio, F. L. H. Tissot, M. Hu, J. Zhao, L. L. Gao, and R. V. Morris (2012), A general moment NRIXS approach to the determination of equilibrium $\mathrm{Fe}$ isotopic fractionation factors: application to goethite and jarosite, Geochim. Cosmochim. Acta, 94, 254-275, doi:10.1016/j.gca.2012.06.013. 
Dewaele, A., P. Loubeyre, F. Occelli, M. Mezouar, P. I. Dorogokupets, and M. Torrent (2006), Quasihydrostatic equation of state of iron above 2 Mbar, Phys. Rev. Lett., 97(21), 4, doi:10.1103/PhysRevLett.97.215504

Dubrovinsky, L. S., S. K. Saxena, and P. Lazor (1998), High-pressure and high-temperature in situ X-ray diffraction study of iron and corundum to $68 \mathrm{GPa}$ using an internally heated diamond anvil cell, Phys. Chem. Miner., 25(6), 434-441.

Dubrovinsky, L. S., S. K. Saxena, F. Tutti, S. Rekhi, and T. LeBehan (2000), In situ X-ray study of thermal expansion and phase transition of iron at multimegabar pressure, Phys. Rev. Lett., 84(8), 1720-1723, doi:10.1103/PhysRevLett.84.1720.

Duffy, T. S., and T. J. Ahrens (1993), Thermal-expansion of mantle and core materials at very high-pressures, Geophys. Res. Lett., 20(11), 1103-1106, doi:10.1029/93gl00479.

Dziewonski, A. M., and D. L. Anderson (1981), Preliminary Reference Earth Model, Phys. Earth Planet. Inter., 25(4), 297-356.

Fiquet, G., J. Badro, F. Guyot, H. Requardt, and M. Krisch (2001), Sound velocities in iron to 110 gigapascals, Science, 291(5503), 468-471.

Fiquet, G., J. Badro, A. Auzende, E. Gregoryanz, J. Siebert, J. Matas, and N. Guignot (2007), A new thermal equation of state for iron at megabar pressure. Eos Trans. AGU 88 (52) Fall Meet. Suppl., Abstract DI24A-05.

Fiquet, G., J. Badro, E. Gregoryanz, Y. W. Fei, and F. Occelli (2009), Sound velocity in iron carbide $(\mathrm{Fe} 3 \mathrm{C})$ at high pressure: Implications for the carbon content of the Earth's inner core, Phys. Earth Planet. Inter. 172(1-2), 125-129, doi:10.1016/j.pepi.2008.05.016.

Gao, L. L., B. Chen, M. Lerche, E. E. Alp, W. Sturhahn, J. Y. Zhao, H. Yavas, and J. Li (2009), Sound velocities of compressed Fe3C from simultaneous synchrotron X-ray diffraction and nuclear resonant scattering measurements, J. Synchrotron Radiat., 16, 714-722, doi:10.1107/ s0909049509033731.

Giefers, H., R. Lübbers, K. Rupprecht, G. Wortmann, D. Alfè, and A. I. Chumakov (2002), Phonon spectroscopy of oriented hcp iron, High Pressure Res., 22(2), 501-506, doi:10.1080/08957950290011437.

Gilvarry, J. J. (1956a), The Lindemann and Grüneisen Laws, Phys. Rev., $102(2), 308-316$.

Gilvarry, J. J. (1956b), Variation of the Amplitude of Thermal Vibration on the Fusion Curve, Phys. Rev., 104(4), 908-913.

Hirao, N., T. Kondo, E. Ohtani, K. Takemura, and T. Kikegawa (2004), Compression of iron hydride to $80 \mathrm{GPa}$ and hydrogen in the Earth's inner core, Geophys. Res. Lett., 31(6), L06616, doi:10.1029/2003g1019380.

Hu, M. Y., W. Sturhahn, T. S. Toellner, P. D. Mannheim, D. E. Brown, J. Y. Zhao, and E. E. Alp (2003), Measuring velocity of sound with nuclear resonant inelastic x-ray scattering, Phys. Rev. B, 67(9), 5, doi:10.1103/PhysRevB.67.094304.

Huang, H. J., Y. W. Fei, L. C. Cai, F. Q. Jing, X. J. Hu, H. S. Xie, L. M. Zhang, and Z. Z. Gong (2011), Evidence for an oxygen-depleted liquid outer core of the Earth, Nature, 479(7374), 513-516, doi:10.1038/ nature10621.

Isaak, D. G., and O. L. Anderson (2003), Thermal expansivity of HCP iron at very high pressure and temperature, Physica B, 328(3-4), 345-354 doi:10.1016/s0921-4526(02)01858-6.

Jackson, J. M., W. Sturhahn, M. Lerche, J. Zhao, T. S. Toellner, E. E. Alp, S. V. Sinogeikin, J. D. Bass, C. A. Murphy, and J. K. Wicks (2013), Melting of compressed iron by monitoring atomic dynamics, Earth Planet. Sci. Lett., 362, 143-150, doi:10.1016/j.eps1.2012.11.048.

Jeanloz, R. (1979), Properties of Iron at High Pressures and the State of the Core, J. Geophys. Res., 84(NB11), 6059-6069, doi:10.1029/ JB084iB11p06059.

Kantor, A. P., I. Y. Kantor, A. V. Kurnosov, A. Y. Kuznetsov, N. A. Dubrovinskaia, M. Krisch, A. A. Bossak, V. P. Dmitriev, V. S. Urusov, and L. S. Dubrovinsky (2007), Sound wave velocities of fcc Fe-Ni alloy at high pressure and temperature by mean of inelastic X-ray scattering, Phys. Earth Planet. Inter., 164(1-2), 83-89, doi:10.1016/j.pepi.2007.06.006.

Kennett, B. L. N., E. R. Engdahl, and R. Buland (1995), Constraints on Seismic Velocities in the Earth from Traveltimes, Geophys. J. Int. 122(1), 108-124, doi:10.1111/j.1365-246X.1995.tb03540.X

Komabayashi, T., and Y. W. Fei (2010), Internally consistent thermodynamic database for iron to the Earth's core conditions, J. Geophys. Res.Solid Earth, 115, 12, B03202, doi:10.1029/2009jb006442.

Lin, J. F., A. J. Campbell, and D. L. Heinz (2003a), Static compression of iron-silicon alloys: Implications for silicon in the Earth's core, J. Geophys. Res., 108(B1), 2045, doi:10.1029/2002JB001978.

Lin, J. F., V. V. Struzhkin, W. Sturhahn, E. Huang, J. Y. Zhao, M. Y. Hu, E. E. Alp, H. K. Mao, N. Boctor, and R. J. Hemley (2003b), Sound velocities of iron-nickel and iron-silicon alloys at high pressures, Geophys. Res. Lett., 30(21), 4, doi:10.1029/2003gl018405.

Lin, J. F., Y. W. Fei, W. Sturhahn, J. Y. Zhao, H. K. Mao, and R. J. Hemley (2004), Magnetic transition and sound velocities of Fe3S at high pressure: implications for Earth and planetary cores, Earth Planet. Sci. Lett., 226(1-2), 33-40, doi:10.1016/j.epsl.2004.07.018.

Lin, J. F., W. Sturhahn, J. Y. Zhao, G. Shen, H. K. Mao, and R. J. Hemley (2005), Sound velocities of hot dense iron: Birch's law revisited, Science, 308(5730), 1892-1894, doi:10.1126/science.1111724.

Lin, J. F., Z. Mao, H. Yavas, J. Y. Zhao, and L. Dubrovinsky (2010), Shear Wave anisotropy of textured hcp-Fe in the Earth's inner core, Earth Planet. Sci. Lett., 298(3-4), 361-366, doi:10.1016/j.eps1.2010.08.006.

Lübbers, R., H. F. Grünsteudel, A. I. Chumakov, and G. Wortmann (2000), Density of phonon states in iron at high pressure, Science, 287(5456), 1250-1253.

Ma, Y. Z., M. Somayazulu, G. Shen, H. K. Mao, J. F. Shu, and R. J. Hemley (2004), In situ X-ray diffraction studies of iron to Earth-core conditions, Phys. Earth Planet. Inter., 143, 455-467, doi:10.1016/j.pepi.2003.06.005.

Mao, H. K., Y. Wu, L. C. Chen, J. F. Shu, and A. P. Jephcoat (1990), Static Compression of Iron to $300 \mathrm{GPa}$ and $\mathrm{Fe}_{0.8} \mathrm{Ni}_{0.2}$ Alloy to $260 \mathrm{GPa}$ : Implications for Composition of the Core, J. Geophys. Res.-Solid Earth Planets, 95(B13), 21737-21742, doi:10.1029/JB095iB13p21737.

Mao, H. K. et al. (2001), Phonon density of states of iron up to 153 gigapascals, Science, 292(5518), 914-916.

Mao, W. L., W. Sturhahn, D. L. Heinz, H. K. Mao, J. F. Shu, and R. J. Hemley (2004), Nuclear resonant X-ray scattering of iron hydride at high pressure, Geophys. Res. Lett., 31(15), L15618, doi:10.1029/2004g1020541.

Mao, W. L., et al. (2008), Experimental determination of the elasticity of iron at high pressure, J. Geophys. Res.-Solid Earth, 113(B9), B09213, doi:10.1029/2007jb005229.

Mao, Z., J. F. Lin, J. Liu, A. Alatas, L. L. Gao, J. Zhao, and H. K. Mao (2012), Sound velocities of Fe and Fe-Si alloy in the Earth's core, Proc. Natl. Acad. Sci., 109(26), 10239-10244, doi:10.1073/pnas.1207086109.

McDonough, W. F. (2003), Compositional Model for the Earth's Core, in Treatise on Geochemistry, The Mantle and Core, edited by D. H. Heinrich and K. T. Karl, pp. 547-568, Pergamon, Oxford.

Murphy, C. A., J. M. Jackson, W. Sturhahn, and B. Chen (2011a), Grüneisen Parameter of hcp-Fe to $171 \mathrm{GPa}$, Geophys. Res. Lett., 38, L24306, doi:10.1029/2011GL049531.

Murphy, C. A., J. M. Jackson, W. Sturhahn, and B. Chen (2011b), Melting and thermal pressure of hcp-Fe from the phonon density of states, Phys. Earth Planet. Inter., 188(1-2), 114-120, doi:10.1016/j.pepi.2011.07.001.

Musgrave, M. J. P. (1970), Crystal Acoustics: Introduction to the Study of Elastic Waves and Vibrations in Crystals, 72 pp., Holden-Day, Inc., San Francisco, Calif.

Nguyen, J. H., and N. C. Holmes (2004), Melting of iron at the physical conditions of the Earth's core, Nature, 427(6972), 339-342, doi:10.1038/ nature 02248 .

Polyakov, V. B. (1998), On anharmonic and pressure corrections to the equilibrium isotopic constants for minerals, Geochim. Cosmochim. Acta, 62(18), 3077-3085, doi:10.1016/s0016-7037(98)00220-8.

Polyakov, V. B., and S. D. Mineev (1999), Mössbauer spectroscopy as applied to isotopic geochemistry: I. Equilibrium fractionation of iron isotopes, Geokhimiya, (8), 858-870.

Polyakov, V. B. (2009), Equilibrium Iron Isotope Fractionation at Core-Mantle Boundary Conditions, Science, 323(5916), 912-914, doi:10.1126/science.1166329.

Prescher, C., L. Dubrovinsky, C. McCammon, K. Glazyrin, Y. Nakajima A. Kantor, M. Merlini, and M. Hanfland (2012), Structurally hidden magnetic transitions in Fe3C at high pressures, Phys. Rev. B, 85(14), doi:10.1103/PhysRevB.85.140402.

Schauble, E. A. (2004), Applying stable isotope fractionation theory to new systems, in Geochemistry of Non-Traditional Stable Isotopes edited by C. M. Johnson, et al., pp. 65-111, Mineralogical Soc Amer, Chantilly.

Scott, H. P., Q. Williams, and E. Knittle (2001), Stability and equation of state of $\mathrm{Fe} 3 \mathrm{C}$ to $73 \mathrm{GPa}$ : Implications for carbon in the Earth's core Geophys. Res. Lett., 28(9), 1875-1878, doi:10.1029/2000gl012606.

Sha, X. W., and R. E. Cohen (2010), First-principles thermal equation of state and thermoelasticity of hcp Fe at high pressures, Phys. Rev. B, 81(9), 10, doi:10.1103/PhysRevB.81.094105.

Shearer, P., and G. Masters (1990), The density and shear velocity contrast at the inner core boundary, Geophys. J. Int., 102(2), 491-498, doi:10.1111/j.1365-246X.1990.tb04481.X

Shen, G., H. K. Mao, R. J. Hemley, T. S. Duffy, and M. L. Rivers (1998), Melting and crystal structure of iron at high pressures and temperatures, Geophys. Res. Lett., 25(3), 373-376, doi:10.1029/97GL03776.

Shibazaki, Y. et al. (2012), Sound velocity measurements in dhcp-FeH up to $70 \mathrm{GPa}$ with inelastic X-ray scattering: Implications for the composition of the Earth's core, Earth Planet. Sci. Lett., 313, 79-85, doi:10.1016/ j.epsl.2011.11.002.

Sola, E., and D. Alfè (2009), Melting of Iron under Earth's Core Conditions from Diffusion Monte Carlo Free Energy Calculations, Phys. Rev. Lett. 103(7), 4, doi:10.1103/PhysRevLett.103.078501. 


\section{MURPHY ET AL.: THERMOELASTICITY OF hcp-Fe TO $171 \mathrm{GPa}$}

Sola, E., J. P. Brodholt, and D. Alfè (2009), Equation of state of hexagonal closed packed iron under Earth's core conditions from quantum Monte Carlo calculations, Phys. Rev. B, 79(2), 6, doi:10.1103/ PhysRevB.79.024107.

Steinle-Neumann, G., L. Stixrude, and R. E. Cohen (2003), Physical properties of iron in the inner core, in Earth's Core: Dynamics, Structure, Rotation, edited by V. Dehant, et al., pp. 137-161, Amer Geophysical Union, Washington.

Steinle-Neumann, G., L. Stixrude, and R. E. Cohen (2004), First-principles elastic constants for the hcp transition metals $\mathrm{Fe}, \mathrm{Co}$, and $\mathrm{Re}$ at high pressure (vol 60, pg 791, 1999), Phys. Rev. B, 69(21), doi:10.1103/ PhysRevB.69.219903.

Stixrude, L., E. Wasserman, and R. E. Cohen (1997), Composition and temperature of Earth's inner core, J. Geophys. Res.-Solid Earth, 102(B11), 24729-24739, doi:10.1029/97JB02125.

Struzhkin, V. V. et al. (2001), Nuclear inelastic X-ray scattering of FeO to 48 GPa, Phys. Rev. Lett., 87(25), doi:10.1103/PhysRevLett.87.255501.

Sturhahn, W., T. S. Toellner, E. E. Alp, X. Zhang, M. Ando, Y. Yoda, S. Kikuta, M. Seto, C. W. Kimball, and B. Dabrowski (1995), Phonon Density of States Measured by Inelastic Nuclear Resonant Scattering, Phys. Rev. Lett., 74(19), 3832-3835.

Sturhahn, W., and A. Chumakov (1999), Lamb-Mössbauer factor and second-order Doppler shift from inelastic nuclear resonant absorption, Hyperfine Interact., 123(1-8), 809-824, doi:10.1023/a:1017060931911.

Sturhahn, W. (2000), CONUSS and PHOENIX: Evaluation of nuclear resonant scattering data, Hyperfine Interact., 125(1-4), 149-172.
Sturhahn, W. (2004), Nuclear resonant spectroscopy, J. Phys.-Condes. Matter, 16(5), S497-S530.

Sturhahn, W., and J. M. Jackson (2007), Geophysical Applications of Nuclear Resonant Spectroscopy, Geol. Soc. Am., Special Paper, 421, 157-174.

Tateno, S., K. Hirose, Y. Ohishi, and Y. Tatsumi (2010), The Structure of Iron in Earth's Inner Core, Science, 330(6002), 359-361, doi:10.1126/ science. 1194662 .

Tateno, S., K. Hirose, T. Komabayashi, H. Ozawa, and Y. Ohishi (2012), The structure of Fe-Ni alloy in Earth's inner core, Geophys. Res. Lett., 39, L12305, doi:10.1029/2012GL052103.

Toellner, T. S. (2000), Monochromatization of synchrotron radiation for nuclear resonant scattering experiments, Hyperfine Interact., 125(1-4), 3-28, doi:10.1023/a:1012621317798.

Vočadlo, L., J. Brodholt, D. P. Dobson, K. S. Knight, W. G. Marshall, G. D. Price, and I. G. Wood (2002), The effect of ferromagnetism on the equation of state of $\mathrm{Fe} 3 \mathrm{C}$ studied by first-principles calculations, Earth Planet. Sci. Lett., 203(1), 567-575, doi:10.1016/s0012-821x(02)00839-7.

Vočadlo, L., D. P. Dobson, and I. G. Wood (2009), Ab initio calculations of the elasticity of hcp-Fe as a function of temperature at inner-core pressure, Earth Planet. Sci. Lett., 288(3-4), 534-538, doi:10.1016/j.eps1.2009.10.015.

Wasserman, E., L. Stixrude, and R. E. Cohen (1996), Thermal properties of iron at high pressures and temperatures, Phys. Rev. B, 53(13), 8296-8309. 